TRANSACTIONS OF THE

AMERICAN MATHEMATICAL SOCIETY

Volume 357, Number 1, Pages 59-88

S 0002-9947(03)03491-3

Article electronically published on December 15, 2003

\title{
LOCAL ZETA FUNCTION FOR CURVES, NON-DEGENERACY CONDITIONS AND NEWTON POLYGONS
}

\author{
M. J. SAIA AND W. A. ZUNIGA-GALINDO
}

\begin{abstract}
This paper is dedicated to a description of the poles of the Igusa local zeta function $Z(s, f, v)$ when $f(x, y)$ satisfies a new non-degeneracy condition called arithmetic non-degeneracy. More precisely, we attach to each polynomial $f(x, y)$ a collection of convex sets $\Gamma^{A}(f)=\left\{\Gamma_{f, 1}, \ldots, \Gamma_{f, l_{0}}\right\}$ called the arithmetic Newton polygon of $f(x, y)$, and introduce the notion of arithmetic non-degeneracy with respect to $\Gamma^{A}(f)$. If $L_{v}$ is a $p$-adic field, and $f(x, y) \in L_{v}[x, y]$ is arithmetically non-degenerate, then the poles of $Z(s, f, v)$ can be described explicitly in terms of the equations of the straight segments that form the boundaries of the convex sets $\Gamma_{f, 1}, \ldots, \Gamma_{f, l_{0}}$. Moreover, the proof of the main result gives an effective procedure for computing $Z(s, f, v)$.
\end{abstract}

\section{INTRODUCTION}

Let $L_{v}$ be a $p$-adic field, and $v$ the corresponding non-archimedean valuation. Let $O_{v}$ the ring of integers of $L_{v}, P_{v}$ the maximal ideal of $O_{v}$, and $\overline{L_{v}}=O_{v} / P_{v}$ the residue field of $L_{v}$. The cardinality of $\overline{L_{v}}$ is denoted by $q$, thus $\overline{L_{v}}=\mathbb{F}_{q}$. We fix a local parameter $\pi$ for $O_{v}$. For $z \in L_{v},|z|_{v}:=q^{-v(z)}$ denotes its normalized absolute value. Given a non-constant polynomial $g(x, y) \in L_{v}[x, y]$, the Igusa local zeta function $Z(s, g, v)$ attached to $g(x, y)$ and $L_{v}$ is defined as

$$
Z(s, g, v):=\int_{O_{v}^{2}}|g(x, y)|_{v}^{s}|d x d y|,
$$

where $s \in \mathbb{C}$ satisfies $\operatorname{Re}(s)>0$ and $|d x d y|$ denotes the Haar measure of $L_{v}^{2}$ normalized so that the measure of $O_{v}^{2}$ is one.

Igusa showed that the local zeta function $Z(s, g, v)$ is a rational function of $q^{-s}$, for general polynomials with coefficients in a $p$-adic field [5]. Igusa's local zeta functions are related to the number of solutions of congruences modulo $p^{m}$ and to exponential sums modulo $p^{m}$. There are several conjectures and intriguing connections between Igusa's local zeta functions with topology and singularity theory [2].

Received by the editors July 10, 2001 and, in revised form, May 6, 2003.

2000 Mathematics Subject Classification. Primary 11D79, 14G20, 14M25.

Key words and phrases. Igusa local zeta functions, Newton polygons, degenerate curves, nondegeneracy conditions, polynomial congruences.

The first named author was partially supported by CNPq-Grant 300556/92-6.

The second named author was supported by COLCIENCIAS-Grant \# 089-2000. The second named author also thanks the partial support given by FAPESP for visiting the Instituto de Matemática e Computaçao, Universidade de São Paulo, Campus São Carlos, in January 2000. 
This paper is dedicated to the description of the poles of the Igusa local zeta functions $Z(s, f, v)$ when $f(x, y)$ satisfies a new non-degeneracy condition called arithmetic non-degeneracy. More precisely, we attach to each polynomial $f(x, y)$ a collection of convex sets

$$
\Gamma^{A}(f)=\left\{\Gamma_{f, 1}, \ldots, \Gamma_{f, l_{0}}\right\}
$$

called the arithmetic Newton polygon of $f(x, y)$, and introduce the notion of arithmetic non-degeneracy with respect to $\Gamma^{A}(f)$ (see Section 4).

The set of degenerate polynomials, in the usual sense, is a proper subset of the set of arithmetically non-degenerate polynomials. In addition, for a fixed geometric Newton polygon $\Gamma^{\text {geom }} \subset \mathbb{R}^{2}$, the set of degenerate complex polynomials in two variables with respect to $\Gamma^{g e o m}$ contains an open set, in the Zariski topology, consisting of arithmetically non-degenerate polynomials.

Our main result gives an explicit list of possible poles of $Z(s, f, v)$, when $f(x, y) \in$ $L_{v}[x, y]$ is an arithmetically non-degenerate polynomial, in terms of the equations of the straight segments that form the boundaries of the convex sets $\Gamma_{f, 1}, \ldots, \Gamma_{f, l_{0}}$ (see Theorems 5.1 6.1). Moreover, our proof gives an effective procedure for computing $Z(s, f, v)$.

The notion of non-degeneracy due Kouchnirenko 9] is useful in the computation of Milnor numbers, the number of isolated solutions of polynomial equations, etc. The authors hope that the notion of arithmetic non-degeneracy may be useful for other purposes, like those mentioned above, different from the description of the poles of local zeta functions.

The description of the poles of the local zeta functions in terms of Newton polygons is a well-known fact for non-degenerate polynomials in the sense of Kouchnirenko [13], [10], 3], 4], [16], [17. In the degenerate case there are no previous results showing a relation between the poles of local zeta functions and Newton polygons.

The poles of $Z(s, f, v)$, for a general polynomial $f$ in two variables, can be described explicitly in terms of a resolution of singularities for $f$ (see [14, 15, [11, [7, 12]). The main result of this paper is complementary to the above-mentioned results. Indeed, our main result produces an explicit list of candidates for the poles of $Z(s, f, v)$ without using resolutions of singularities, but this result is only valid for a large class of polynomials.

In this paper, we work with $p$-adic fields of characteristic zero; however all results are valid for positive characteristics.

This paper is organized as follows. In Section 2, we review some well-known results about geometric Newton polygons, non-degeneracy conditions in the sense of Kouchnirenko, and certain $p$-adic integrals attached to non-degenerate polynomials. In Section 3, we compute explicitly the local zeta function of some degenerate polynomials, in the sense of Kouchnirenko. These examples constitute the basic models for the effective computation of the local zeta functions of arithmetically non-degenerate polynomials. In Section 4, we introduce the notion of arithmetic Newton polygon and arithmetic non-degeneracy condition. In Section 5, we compute several $p$-adic integrals that appear in the effective computation of the local zeta function of an arithmetically non-degenerate polynomial. In Section 6, we prove the main result. 


\section{Preliminaries}

2.1. Geometric Newton polygons. We set $\mathbb{R}_{+}=\{x \in \mathbb{R} \mid x \geq 0\}$. Let $f(x, y)=$ $\sum_{(i, j)} a_{i, j} x^{i} y^{j}$ be a non-constant polynomial in two variables with coefficients in a field $K$ satisfying $f(0,0)=0$. The set $\operatorname{supp}(f)=\left\{(i, j) \in \mathbb{N}^{2} \mid a_{i, j} \neq 0\right\}$ is called the support of $f$. The geometric Newton polygon $\Gamma^{g e o m}(f)$ of $f$ is defined as the convex hull in $\mathbb{R}_{+}^{2}$ of the set $\bigcup_{(i, j) \in \operatorname{supp}(f)}\left((i, j)+\mathbb{R}_{+}^{2}\right)$.

By a proper face $\gamma$ of $\Gamma^{g e o m}(f)$, we mean a non-empty convex set $\gamma$ obtained by intersecting $\Gamma^{\text {geom }}(f)$ with a straight line $H$, such that $\Gamma^{\text {geom }}(f)$ is contained in one of the two half-spaces determined by $H$. The straight line $H$ is called the supporting line of $\gamma$. Thus the faces can be points (zero-dimensional faces) or straight segments (one-dimensional faces). The last ones are also called facets.

If $\gamma$ is a face of $\Gamma^{g e o m}(f)$, its face function is defined to be the polynomial

$$
f_{\gamma}(x, y):=\sum_{(i, j) \in \gamma} a_{i, j} x^{i} y^{j} .
$$

Definition 2.1. A non-constant polynomial $f(x, y)$ satisfying $f(0,0)=0$ is called non-degenerate with respect to $\Gamma^{\text {geom }}(f)$, in the sense Kouchnirenko, if it satisfies:

(1) the origin of $K^{2}$ is a singular point of $f(x, y)$;

(2) for each face $\gamma \subseteq \Gamma^{g e o m}(f)$, including $\Gamma^{g e o m}(f)$ itself, the system of equations

$$
f_{\gamma}(x, y)=\frac{\partial f_{\gamma}}{\partial x}(x, y)=\frac{\partial f_{\gamma}}{\partial y}(x, y)=0
$$

does not have solutions on $K^{\times 2}$.

We set $\langle.,$.$\rangle for the usual inner product in \mathbb{R}^{2}$, and identify the dual vector space with $\mathbb{R}^{2}$. For $a \in \mathbb{R}_{+}^{2}$, we define

$$
m(a):=\inf _{x \in \Gamma(f)}\{\langle a, x\rangle\} .
$$

Given any $a \in \mathbb{R}_{+}^{2} \backslash\{0\}$, the first meet locus $F(a)$ of $a$ is defined as

$$
F(a):=\left\{x \in \Gamma^{\text {geom }}(f) \mid\langle a, x\rangle=m(a)\right\} .
$$

The first meet locus $F(a)$ of $a \in \mathbb{R}_{+}^{2} \backslash\{0\}$ is a proper face of $\Gamma^{\text {geom }}(f)$.

Given a proper face $\gamma$ of $\Gamma^{\text {geom }}(f)$, the cone associated to $\gamma$ is defined to be the set

$$
\Delta_{\gamma}:=\left\{a \in \mathbb{R}_{+}^{2} \backslash\{0\} \mid F(a)=\gamma\right\} .
$$

We define an equivalence relation on $\mathbb{R}_{+}^{2} \backslash\{0\}$ by

$$
a \simeq a^{\prime} \text { if and only if } F(a)=F\left(a^{\prime}\right) .
$$

The equivalence classes of $\simeq$ are the cones $\Delta_{\gamma}$. The following two propositions describe the geometry of the equivalence classes of $\simeq$ (see e.g. [13, [3]).

Proposition 2.1. Let $\gamma$ be a proper face of $\Gamma^{g e o m}(f)$. If $\gamma$ is a one-dimensional face and $a$ is a perpendicular vector to $\gamma$, then

$$
\Delta_{\gamma}=\{\alpha a \mid \alpha \in \mathbb{R}, \alpha>0\} .
$$

If $\gamma$ is a zero-dimensional face and $w_{1}, w_{2}$ are the facets of $\Gamma^{g e o m}(f)$ which contain $\gamma$, and $a_{1}, a_{2}$ are vectors which are perpendicular to $w_{1}, w_{2}$, respectively, then

$$
\Delta_{\gamma}=\left\{\alpha_{1} a_{1}+\alpha_{2} a_{2} \mid \alpha_{i} \in \mathbb{R}, \alpha_{i}>0\right\} .
$$


A set of the form (2.2) (respectively (2.1)) is called a strictly positive cone spanned by the vectors $\left\{a_{1}, a_{2}\right\}$ (respectively by $\{a\}$ ). Let $\Delta$ be a strictly positive cone spanned by a vector set $A$, with $A=\left\{a_{1}, a_{2}\right\}$, or $A=\{a\}$. If $A$ is linearly independent over $\mathbb{R}$, the cone $\Delta$ is called a simplicial cone. In this last case, if the elements of $A$ are in $\mathbb{Z}^{2}$, the cone $\Delta$ is called a rational simplicial cone. If $A$ can be completed to be a basis of the $\mathbb{Z}$-module $\mathbb{Z}^{2}$, the cone $\Delta$ is called a simple cone.

A vector $a \in \mathbb{R}^{2}$ is called primitive if the components of $a$ are positive integers whose greatest common divisor is one.

For every facet of $\Gamma^{g e o m}(f)$ there is a unique primitive vector in $\mathbb{R}^{2}$ which is perpendicular to this facet. We denote by $D$ the set of such vectors. Thus each equivalence class under $\simeq$ is a rational simplicial cone spanned by elements of $D$.

From the above considerations it follows that there exists a partition of $\mathbb{R}_{+}^{2}$ of the form

$$
\mathbb{R}_{+}^{2}=\{(0,0)\} \cup \underset{\gamma \subset \Gamma^{\text {geom }}(f)}{\bigcup_{\gamma}}
$$

where $\gamma$ runs through all proper faces of $\Gamma^{\text {geom }}(f)$. We say that $\left\{\Delta_{\gamma}\right\}_{\gamma \subset \Gamma^{\text {geom }}(f)}$ is a simplicial conical subdivision of $\mathbb{R}_{+}^{2}$ subordinated to $\Gamma^{\text {geom }}(f)$.

2.2. Local zeta functions and conical subdivisions. Let $f(x, y) \in L_{v}[x, y]$ be a non-constant polynomial satisfying $f(0,0)=0$, and $\Gamma^{g e o m}(f)$ its geometric Newton polygon. We fix a simplicial conical subdivision $\left\{\Delta_{\gamma}\right\}_{\gamma \subset \Gamma^{\text {geom }}(f)}$ of $\mathbb{R}_{+}^{2}$ subordinated to $\Gamma^{g e o m}(f)$. We define

$$
\begin{gathered}
E_{\Delta_{\gamma}}:=\left\{(x, y) \in O_{v}^{2} \mid(v(x), v(y)) \in \Delta_{\gamma}\right\}, \\
Z\left(s, f, v, \Delta_{\gamma}\right):=\int_{E_{\Delta_{\gamma}}}|f(x, y)|_{v}^{s}|d x d y|,
\end{gathered}
$$

and

$$
Z\left(s, f, v, O_{v}^{\times 2}\right):=\int_{O_{v}^{\times 2}}|f(x, y)|_{v}^{s}|d x d y| .
$$

With the above notation, it follows from partition (2.3) that

$$
Z(s, f, v)=Z\left(s, f, v, O_{v}^{\times 2}\right)+\sum_{\gamma \subset \Gamma^{\text {geom }}(f)} Z\left(s, f, v, \Delta_{\gamma}\right) .
$$

Therefore the computation of the integral $Z(s, f, v)$ is reduced to the computation of integrals of the type $Z\left(s, f, v, O_{v}^{\times 2}\right)$ and $Z\left(s, f, v, \Delta_{\gamma}\right)$. In the case in which $f$ is non-degenerate with respect to $\Gamma^{g e o m}(f)$, the above-mentioned integrals can be computed explicitly using techniques of toroidal geometry and the $p$-adic stationary phase formula [5. Theorem 10.2.1], [4, [16], 17], [18].

For a general $f$ formula (2.4) is still valuable if $f$ does not have singularities on $\left(L_{v}^{\times}\right)^{2}$. By applying the stationary phase formula (abbreviated SPF) repeatedly, it is possible to compute $Z\left(s, f, v, O_{v}^{\times 2}\right)$ effectively (see [16, Lemma 3.1], or [17. Lemma 2.4]). For a future reference we restate this result here.

Lemma 2.1. Let $g_{m}(x, y)=g_{0}(x, y)+\pi^{m} g_{1}(x, y) \in L_{v}[x, y]$ be a non-constant polynomial, $m \geq 1$, such that $g_{m}(x, y)$ and $g_{0}(x, y)$ do not have singular points on 
$\left(L_{v}^{\times}\right)^{2}$. There exists a constant $c\left(g_{0}\right)$ such that if $m \geq c\left(g_{0}\right)$, then

$$
Z\left(s, g_{m}, v, O_{v}^{\times 2}\right)=\int_{O_{v}^{\times 2}}\left|g_{0}(x, y)\right|_{v}^{s}|d x d y|=\frac{U\left(q^{-s}\right)}{1-q^{-1-s}},
$$

with $U\left(q^{-s}\right) \in \mathbb{Q}\left[q^{-s}\right]$.

The computation of the integrals $Z\left(s, f, v, \Delta_{\gamma}\right)$ presents two cases. If $f_{\gamma}$ does not have singularities on $\left(L_{v}^{\times}\right)^{2}$, we shall say that $f$ is non-degenerate on $\gamma$. In this case the integrals $Z\left(s, f, v, \Delta_{\gamma}\right)$ can be computed by using known techniques [10], [4], [16], [17]. For a future reference we restate this result here.

Lemma 2.2. Let $f(x, y) \in L_{v}[x, y]$ be a non-constant polynomial such that $f(0,0)$ $=0$ and $f$ does not have singular points on $\left(L_{v}^{\times}\right)^{2}$.

(1) If $\Delta_{\gamma}$ is a two-dimensional cone, and $f_{\gamma}(x, y)$ does not have singularities on the torus $\left(L_{v}^{\times}\right)^{2}$, then

$$
Z\left(s, f, v, \Delta_{\gamma}\right)=U_{1}\left(q^{-s}\right) \in \mathbb{Q}\left[q^{-s}\right] .
$$

(2) If $\Delta_{\gamma}$ is a one-dimensional cone, and $f_{\gamma}(x, y)$ does not have singularities on $\left(L_{v}^{\times}\right)^{2}$, then

$$
Z\left(s, f, v, \Delta_{\gamma}\right)=\frac{U_{2}\left(q^{-s}\right)}{\left(1-q^{-1-s}\right)\left(1-q^{-(a+b)-d_{\gamma} s}\right)}, \quad U_{2}\left(q^{-s}\right) \in \mathbb{Q}\left[q^{-s}\right],
$$

where $a x+b y=d_{\gamma}$ is the equation of the supporting line of the facet $\gamma$.

The core of this paper is the explicit computation of integrals of the type

$$
Z\left(s, f, v, \Delta_{\gamma}\right) \text {, }
$$

when $f$ is degenerate on $\Delta_{\gamma}$, but satisfies a new non-degeneracy condition. This will be done in Sections 4, 5 .

Remark 2.1. Given $\Delta$ a rational simplicial cone, there exists a partition of $\Delta$ into simple cones [8, pp. 32-33]. This fact can be used to obtain a partition of $\mathbb{R}_{+}^{2}$ into simple cones. In this case, it is possible to compute the integral $Z(s, f, v, \Delta)$ in a simple form, when $f$ is non-degenerate, in the sense of Kouchnirenko. However, this procedure produces a larger list of candidates for poles of $Z(s, f, v, \Delta)$.

\section{MODEL CASES}

In this section we shall compute explicitly the local zeta functions for some degenerate polynomials, in the sense of Kouchnirenko. These examples constitute the basic models for the effective computation of the local zeta functions of arithmetically non-degenerate polynomials.

3.1. The local zeta function of $\left(y^{3}-x^{2}\right)^{2}+x^{4} y^{4}$. We assume that the characteristic of the residue field of $L_{v}$ is different from 2. The origin of $L_{v}^{2}$ is the only singular point of $f(x, y)=\left(y^{3}-x^{2}\right)^{2}+x^{4} y^{4}$. This polynomial is degenerate with respect to its geometric Newton polygon.

The conical subdivision of $\mathbb{R}_{+}^{2}$ subordinated to the geometric Newton polygon of $f(x, y)$ is

$$
\mathbb{R}_{+}^{2}=\{(0,0)\} \cup \bigcup_{j=1}^{9} \Delta_{j},
$$


with

\begin{tabular}{|l|}
\hline$\Delta_{1}:=(0,1) \mathbb{R}_{+}$ \\
\hline$\Delta_{2}:=(0,1) \mathbb{R}_{+}+(1,1) \mathbb{R}_{+}$ \\
\hline$\Delta_{3}:=(1,1) \mathbb{R}_{+}$ \\
\hline$\Delta_{4}:=(1,1) \mathbb{R}_{+}+(3,2) \mathbb{R}_{+}$ \\
\hline$\Delta_{5}=:(3,2) \mathbb{R}_{+}$ \\
\hline$\Delta_{6}:=(3,2) \mathbb{R}_{+}+(2,1) \mathbb{R}_{+}$ \\
\hline$\Delta_{7}:=(2,1) \mathbb{R}_{+}$ \\
\hline$\Delta_{8}:=(2,1) \mathbb{R}_{+}+(1,0) \mathbb{R}_{+}$ \\
\hline$\Delta_{9}:=(1,0) \mathbb{R}_{+}$ \\
\hline
\end{tabular}

In addition, all the $\Delta_{i}$ are rational simple cones. By using the above subdivision it is possible to reduce the computation of $Z(s, f, v)$ to the computation of $Z\left(s, f, v, O_{v}^{\times 2}\right)$ and $Z\left(s, f, v, \Delta_{i}\right), i=1,2,3,4,6,7,8,9$ (see [2.2).

3.2. Computation of $Z\left(s, f, v, O_{v}^{\times 2}\right)$. Since $f(x, y)$ does not have singularities on $\left(L_{v}^{\times}\right)^{2}$, the integral can be computed by using SPF [5. Theorem 10.2.1]:

$$
Z\left(s, f, v, O_{v}^{\times 2}\right)=\left[\left(1-q^{-1}\right)^{2}-q^{-2} N(f)\right]+\frac{q^{-2}(N(f)-1)\left(1-q^{-1}\right) q^{-s}}{1-q^{-1-s}},
$$

with $N(f)=\operatorname{Card}\left(\left\{(u, v) \in\left(\mathbb{F}_{q}^{\times}\right)^{2} \mid f(u, v)=0\right\}\right)$.

3.3. Computation of $Z\left(s, f, v, \Delta_{i}\right), i=1,2,3,4,6,7,8,9$. These integrals correspond to the case in which $f$ is non-degenerate on $\Delta_{i}, i=1,2,3,4,6,7,8,9$. The integrals corresponding to $\Delta_{i}, i=1,2$, can be calculated as follows.

3.3.1. Case $Z\left(s, f, v, \Delta_{1}\right)$.

$$
\begin{aligned}
Z\left(s, f, v, \Delta_{1}\right) & =\sum_{n=1}^{\infty} \int_{O_{v}^{\times} \times \pi^{n} O_{v}^{\times}}|f(x, y)|_{v}^{s}|d x d y| \\
& =\sum_{n=1}^{\infty} q^{-n} \int_{O_{v}^{\times 2}}\left|\left(\pi^{3 n} y^{3}-x^{2}\right)^{2}+\pi^{4 n} x^{4} y^{4}\right|_{v}^{s}|d x d y| \\
& =\left(1-q^{-1}\right)^{2} \sum_{n=1}^{\infty} q^{-n}=q^{-1}\left(1-q^{-1}\right) .
\end{aligned}
$$

3.3.2. Case $Z\left(s, f, v, \Delta_{2}\right)$.

$$
\begin{gathered}
Z\left(s, f, v, \Delta_{2}\right)=\sum_{m=1}^{\infty} \sum_{n=1}^{\infty} \int_{\pi^{m} O_{v}^{\times} \times \pi^{n+m} O_{v}^{\times}}|f(x, y)|_{v}^{s}|d x d y| \\
=\sum_{m=1}^{\infty} \sum_{n=1}^{\infty} q^{-2 m-n-4 m s} \int_{O_{v}^{\times 2}}\left|\left(\pi^{3 n+m} y^{3}-x^{2}\right)^{2}+\pi^{4 m+4 n} x^{4} y^{4}\right|_{v}^{s}|d x d y| \\
=\left(1-q^{-1}\right) q^{-1} \frac{q^{-2-4 s}}{\left(1-q^{-2-4 s}\right)} .
\end{gathered}
$$


The other integrals can be computed in the same form. The following table summarizes these computations:

\begin{tabular}{|l|l|}
\hline Cone & $Z(s, f, v, \Delta)$ \\
\hline$\Delta_{1}$ & $q^{-1}\left(1-q^{-1}\right)$ \\
\hline$\Delta_{2}$ & $\left(1-q^{-1}\right) q^{-1} \frac{q^{-2-4 s}}{\left(1-q^{-2-4 s}\right)}$ \\
\hline$\Delta_{3}$ & $\left(1-q^{-1}\right)^{2} \frac{q^{-2-4 s}}{\left(1-q^{-2-4 s}\right)}$ \\
\hline$\Delta_{4}$ & $\left(1-q^{-1}\right)^{2} \frac{q^{-7-16 s}}{\left(1-q^{-2-4 s}\right)\left(1-q^{-5-12 s}\right)}$ \\
\hline$\Delta_{6}$ & $\frac{\left(1-q^{-1}\right)^{2} q^{-8-18 s}}{\left(1-q^{-5-12 s}\right)\left(1-q^{-3-6 s}\right)}$ \\
\hline$\Delta_{7}$ & $\frac{\left(1-q^{-1}\right)^{2} q^{-3-6 s}}{\left(1-q^{-3-6 s}\right)}$ \\
\hline$\Delta_{8}$ & $\frac{\left(1-q^{-1}\right) q^{-4-6 s}}{\left(1-q^{-3-6 s}\right)}$ \\
\hline$\Delta_{9}$ & $q^{-1}\left(1-q^{-1}\right)$ \\
\hline
\end{tabular}

3.4. Computation of $Z\left(s, f, v, \Delta_{5}\right)$ (an integral on a degenerate face in the sense of Kouchnirenko).

$$
\begin{aligned}
Z\left(s, f, v, \Delta_{5}\right) & =\sum_{n=1}^{\infty} \int_{\pi^{3 n} O_{v}^{\times} \times \pi^{2 n} O_{v}^{\times}}|f(x, y)|_{v}^{s}|d x d y| \\
& =\sum_{n=1}^{\infty} q^{-5 n-12 n s} \int_{O_{v}^{\times 2}}\left|\left(y^{3}-x^{2}\right)^{2}+\pi^{8 n} x^{4} y^{4}\right|_{v}^{s}|d x d y| .
\end{aligned}
$$

We set $f^{(n)}(x, y)=\left(y^{3}-x^{2}\right)^{2}+\pi^{8 n} x^{4} y^{4}$, for $n \geq 1$. In order to compute (3.4), we first compute the integral

$$
I\left(s, f^{(n)}, v\right):=\int_{O_{v}^{\times 2}}\left|\left(y^{3}-x^{2}\right)^{2}+\pi^{8 n} x^{4} y^{4}\right|_{v}^{s}|d x d y|, n \geq 1 .
$$

For that, we use the following change of variables:

$$
\Phi: \begin{gathered}
O_{v}^{\times 2} \\
(x, y)
\end{gathered} \longrightarrow \begin{gathered}
O_{v}^{\times 2} \\
\left(x^{3} y, x^{2} y\right) .
\end{gathered}
$$

The map $\Phi$ gives an analytic bijection of $O_{v}^{\times 2}$ onto itself and preserves the Haar measure because its Jacobian $J_{\Phi}(x, y)=x^{4} y$ satisfies $\left|J_{\Phi}(x, y)\right|_{v}=1$, for every $x, y \in O_{v}^{\times}$. Thus

$$
f^{(n)} \circ \Phi(x, y)=x^{12} y^{4} \widetilde{f^{(n)}}(x, y)
$$

with

$$
\widetilde{f^{(n)}}(x, y):=(y-1)^{2}+\pi^{8 n} x^{8} y^{4}
$$

and then from (3.6), and (3.7), it follows that

$$
I\left(s, f^{(n)}, v\right)=\int_{O_{v}^{\times 2}}\left|\widetilde{f^{(n)}}(x, y)\right|_{v}^{s}|d x d y| .
$$


In order to compute the integral $I\left(s, f^{(n)}, v\right), n \geq 1$, we decompose $O_{v}^{\times 2}$ as follows:

$$
O_{v}^{\times 2}=\bigcup_{y_{0} \not \equiv 1 \bmod \pi} O_{v}^{\times} \times\left\{y_{0}+\pi O_{v}\right\} \cup O_{v}^{\times} \times\left\{1+\pi O_{v}\right\}
$$

where $y_{0}$ runs through a set of representatives of $\mathbb{F}_{q}^{\times}$in $O_{v}$. From partition (3.10) and formula (3.8), it follows that

$$
I\left(s, f^{(n)}, v\right)=(q-2) q^{-1}\left(1-q^{-1}\right)+\int_{O_{v}^{\times} \times\left\{1+\pi O_{v}\right\}}\left|\widetilde{f^{(n)}}(x, y)\right|_{v}^{s}|d x d y| .
$$

The integral on the right side of (3.11) admits the following expansion:

$$
I\left(s, f^{(n)}, v\right)=(q-2) q^{-1}\left(1-q^{-1}\right)+\sum_{j=1}^{\infty} q^{-j} \int_{O_{v}^{\times 2}}\left|\widetilde{f^{(n)}}\left(x, 1+\pi^{j} z\right)\right|_{v}^{s}|d x d z|
$$

where $\widetilde{f^{(n)}}\left(x, 1+\pi^{j} z\right)=\pi^{2 j} z^{2}+\pi^{8 n} x^{8}\left(1+\pi^{j} z\right)^{4}$. In the next step, we compute $\left|\widetilde{f^{(n)}}\left(x, 1+\pi^{j} z\right)\right|_{v}^{s}$ explicitly, for a fixed $n \geq 1$. In this case, this can be done by a simple calculation:

$$
\left|\widetilde{f^{(n)}}\left(x, 1+\pi^{j} z\right)\right|_{v}^{s}= \begin{cases}q^{-2 j s} & \text { if } 1 \leq j \leq 4 n-1 \\ q^{-8 n s}\left|z^{2}+x^{8}\left(1+\pi^{j} z\right)^{4}\right|_{v}^{s} & \text { if } j=4 n \\ q^{-8 n s} & \text { if } j \geq 4 n+1\end{cases}
$$

for any $(x, z) \in O_{v}^{\times 2}$.

We note that the explicit computation of the absolute value $\left|\widetilde{f^{(n)}}\left(x, 1+\pi^{j} z\right)\right|_{v}$ depends on an explicit description of the set $\left\{(w, z) \in \mathbb{R}^{2} \mid w \leq \min \{2 z, 8 n\}\right\}$, for a fixed $n$.

Now from (3.12), 3.13), and the identity $\sum_{k=A}^{B} z^{k}=\frac{z^{A}-z^{B+1}}{1-z}$, we obtain an explicit expression for $I\left(s, f_{n}, v\right)$ :

$$
\begin{aligned}
I\left(s, f^{(n)}, v\right)= & (q-2) q^{-1}\left(1-q^{-1}\right)+\left(1-q^{-1}\right)^{2} \frac{q^{-1-2 s}}{\left(1-q^{-1-2 s}\right)} \\
& -\left(1-q^{-1}\right)^{2} \frac{q^{-4 n-8 n s}}{\left(1-q^{-1-2 s}\right)}+q^{-4 n-8 n s} I_{0}(s) \\
& +q^{-1}\left(1-q^{-1}\right) q^{-4 n-8 n s},
\end{aligned}
$$

where

$$
I_{0}(s):=\int_{O_{v}^{\times 2}}\left|z^{2}+x^{8}\left(1+\pi^{4 n} z\right)^{4}\right|_{v}^{s}|d x d z|
$$

Since the reduction modulo $\pi$ of the polynomial $z^{2}+x^{8}\left(1+\pi^{4 n} z\right)^{4}$ does not have singular points on $\mathbb{F}_{q}^{\times 2}$, because the characteristic of the residue field is different from 2, SPF implies that $I_{0}(s)=\frac{U_{1}\left(q^{-s}\right)}{\left(1-q^{-1-s}\right)}$, where $U_{1}\left(q^{-s}\right)$ is a polynomial in $q^{-s}$ independent of $j$ and $n$. 
Finally, from (3.4), (3.9) and (3.14), we obtain the following explicit expression for $Z\left(s, f, v, \Delta_{5}\right)$ :

$$
\begin{aligned}
Z\left(s, f, v, \Delta_{5}\right)= & \frac{(q-2)\left(1-q^{-1}\right) q^{-6-12 s}}{\left(1-q^{-5-12 s}\right)}+\frac{\left(1-q^{-1}\right)^{2} q^{-6-14 s}}{\left(1-q^{-1-2 s}\right)\left(1-q^{-5-12 s}\right)} \\
& -\frac{\left(1-q^{-1}\right)^{2} q^{-9-20 s}}{\left(1-q^{-1-2 s}\right)\left(1-q^{-9-20 s}\right)}+\frac{q^{-9-20 s} U_{1}\left(q^{-s}\right)}{\left(1-q^{-1-s}\right)\left(1-q^{-9-20 s}\right)} \\
& +\frac{\left(1-q^{-1}\right) q^{-10-20 s}}{\left(1-q^{-9-20 s}\right)} .
\end{aligned}
$$

From the explicit formulas for $Z\left(s, f, v, \Delta_{i}\right), i=1,2, \ldots, 8$, and $Z\left(s, f, v, O_{v}^{\times 2}\right)$ (see (3.3), (3.16)), it follows that the real parts of the poles of $Z(s, f, v)$ belong to the set

$$
\{-1\} \cup\left\{-\frac{5}{12}\right\} \cup\left\{-\frac{1}{2},-\frac{9}{20}\right\} .
$$

3.5. The local zeta function of $\left(y^{3}-x^{2}\right)^{2}\left(y^{3}-a x^{2}\right)+x^{4} y^{4}$. We set $g(x, y)=$ $\left(y^{3}-x^{2}\right)^{2}\left(y^{3}-a x^{2}\right)+x^{4} y^{4} \in L_{v}[x, y]$, with $a \in O_{v}^{\times}$, and $a \not \equiv 1 \bmod \pi$. We assume that the characteristic of the residue field of $L_{v}$ is different from 2 . The polynomial $g$ is degenerate with respect to its geometric Newton polygon. In addition, the origin of $L_{v}^{2}$ is the only singular point of $g$.

By using the same decomposition of $\mathbb{R}_{+}^{2}$ into rational simple cones as in the above example, it is possible to reduce the computation of $Z(s, g, v)$ to the computation of the $p$-adic integrals $Z\left(s, g, v, O_{v}^{\times 2}\right), Z\left(s, g, v, \Delta_{i}\right), i=1, \ldots, 8,9$.

3.6. Computation of $Z\left(s, g, v, O_{v}^{\times 2}\right)$. Since $g(x, y)$ does not have singularities on $\left(L_{v}^{\times}\right)^{2}$, the integral can be computed by using SPF [5, Theorem 10.2.1]:

$$
Z\left(s, g, v, O_{v}^{\times 2}\right)=\left[\left(1-q^{-1}\right)^{2}-q^{-2} N(g)\right]+\frac{q^{-2}(N(g)-1)\left(1-q^{-1}\right) q^{-s}}{1-q^{-1-s}},
$$

with $N(g)=\operatorname{Card}\left(\left\{(u, v) \in\left(\mathbb{F}_{q}^{\times}\right)^{2} \mid g(u, v)=0\right\}\right)$.

3.7. Computation of $Z\left(s, g, v, \Delta_{i}\right), i=1,2,3,4,6,7,8,9$. These integrals can be computed as the corresponding integrals in the previous example. The following table summarizes these computations.

\begin{tabular}{|l|l|}
\hline Cone & $Z(s, g, v, \Delta)$ \\
\hline$\Delta_{1}$ & $q^{-1}\left(1-q^{-1}\right)$ \\
\hline$\Delta_{2}$ & $\left(1-q^{-1}\right) \frac{q^{-3-6 s}}{\left(1-q^{-2-6 s}\right)}$ \\
\hline$\Delta_{3}$ & $\left(1-q^{-1}\right)^{2} \frac{q^{-2-6 s}}{\left(1-q^{-2-6 s}\right)}$ \\
\hline$\Delta_{4}$ & $\left(1-q^{-1}\right)^{2} \frac{q^{-7-24 s}}{\left(1-q^{-2-6 s}\right)\left(1-q^{-5-18 s}\right)}$ \\
\hline$\Delta_{6}$ & $\frac{\left(1-q^{-1}\right)^{2} q^{-8-27 s}}{\left(1-q^{-5-18 s}\right)\left(1-q^{-3-9 s}\right)}$ \\
\hline$\Delta_{7}$ & $\frac{\left(1-q^{-1}\right)^{2} q^{-3-9 s}}{\left(1-q^{-3-9 s}\right)}$ \\
\hline$\Delta_{8}$ & $\frac{\left(1-q^{-1}\right) q^{-4-9 s}}{\left(1-q^{-3-9 s}\right)}$ \\
\hline$\Delta_{9}$ & $q^{-1}\left(1-q^{-1}\right)$ \\
\hline
\end{tabular}


3.8. Computation of $Z\left(s, f, v, \Delta_{5}\right)$ (an integral on a degenerate face in the sense of Kouchnirenko).

$$
\begin{aligned}
Z\left(s, g, v, \Delta_{5}\right) & =\sum_{n=1}^{\infty} \int_{\pi^{3 n} O_{v}^{\times} \times \pi^{2 n} O_{v}^{\times}}|g(x, y)|_{v}^{s}|d x d y| \\
& =\sum_{n=1}^{\infty} q^{-5 n-18 n s} \int_{O_{v}^{\times 2}}\left|\left(y^{3}-x^{2}\right)^{2}\left(y^{3}-a x^{2}\right)+\pi^{2 n} x^{4} y^{4}\right|_{v}^{s}|d x d y| .
\end{aligned}
$$

We set $g^{(n)}(x, y)=\left(y^{3}-x^{2}\right)^{2}\left(y^{3}-a x^{2}\right)+\pi^{2 n} x^{4} y^{4}$, for $n \geq 1$. In order to compute (3.19), we first compute the integral

$$
I\left(s, g^{(n)}, v\right):=\int_{O_{v}^{\times 2}}\left|\left(y^{3}-x^{2}\right)^{2}\left(y^{3}-a x^{2}\right)+\pi^{2 n} x^{4} y^{4}\right|_{v}^{s}|d x d y|, n \geq 1 .
$$

By using the change of variables $\Phi(x, y)$ defined in (3.6), we have that

$$
g^{(n)} \circ \Phi(x, y)=x^{18} y^{6} \widetilde{g^{(n)}}(x, y) \text {, }
$$

with

$$
\widetilde{g^{(n)}}(x, y)=(y-1)^{2}(y-a)+\pi^{2 n} x^{2} y^{2} .
$$

Since $\Phi(x, y)$ gives a bijection of $O_{v}^{\times 2}$ onto itself preserving the Haar measure, it follows from (3.20) and (3.21), that

$$
I\left(s, g^{(n)}, v\right)=\int_{O_{v}^{\times 2}}\left|\widetilde{g^{(n)}}(x, y)\right|_{v}^{s}|d x d y| .
$$

In order to compute the integral $I\left(s, g^{(n)}, v\right)$, we decompose $O_{v}^{\times 2}$ as follows:

$$
\begin{aligned}
O_{v}^{\times 2}= & \left(O_{v}^{\times} \times\left\{y_{0}+\pi O_{v} \mid y_{0} \not \equiv 1, a \bmod \pi\right\}\right) \cup\left(O_{v}^{\times} \times\left\{1+\pi O_{v}\right\}\right) \\
& \cup\left(O_{v}^{\times} \times\left\{a+\pi O_{v}\right\}\right) .
\end{aligned}
$$

From the above partition it follows that

$$
\begin{gathered}
I\left(s, g^{(n)}, v\right)=\left(1-q^{-1}\right) q^{-1}(q-3) \\
+\sum_{j=1}^{\infty} q^{-j} \int_{O_{v}^{\times} \times O_{v}^{\times}}\left|\widetilde{g^{(n)}}\left(x, 1+\pi^{j} y\right)\right|_{v}^{s}|d x d y| \\
+\sum_{j=1}^{\infty} q^{-j} \int_{O_{v}^{\times} \times O_{v}^{\times}}\left|\widetilde{g^{(n)}}\left(x, a+\pi^{j} y\right)\right|_{v}^{s}|d x d y|,
\end{gathered}
$$

with

$$
\begin{gathered}
\widetilde{g^{(n)}}\left(x, 1+\pi^{j} y\right)=\pi^{2 j} \gamma_{1, j}(x, y) y^{2}+\pi^{2 n} \gamma_{2, j}(x, y) x^{2}, \\
\gamma_{1, j}(x, y)=1-a+\pi^{j} y, \quad \gamma_{2, j}(x, y)=\left(1+\pi^{j} y\right)^{2},
\end{gathered}
$$

and

$$
\begin{gathered}
\widetilde{g^{(n)}}\left(x, a+\pi^{j} y\right)=\pi^{j} \delta_{1}(x, y) y+\pi^{2 n} \delta_{2}(x, y) x^{2}, \\
\delta_{1, j}(x, y)=\left(a-1+\pi^{j} y\right)^{2}, \quad \delta_{2, j}(x, y)=\left(a+\pi^{j} y\right)^{2} .
\end{gathered}
$$


In the next step, we explicitly compute $\left.\widetilde{\mid g^{(n)}}\left(x, 1+\pi^{j} y\right)\right|_{v} ^{s}$ and $\left.\widetilde{\mid g^{(n)}}\left(x, a+\pi^{j} y\right)\right|_{v} ^{s}$, for a fixed $n \geq 1$. By some simple calculations,

$$
\left.\widetilde{\mid g^{(n)}}\left(x, 1+\pi^{j} y\right)\right|_{v} ^{s}= \begin{cases}q^{-2 j s} & \text { if } 1 \leq j \leq n-1, \\ q^{-2 n s}\left|\gamma_{1, j}(x, y) y^{2}+\gamma_{2, j}(x, y) x^{2}\right|_{v}^{s} & \text { if } j=n \\ q^{-2 n s} & \text { if } j \geq n+1\end{cases}
$$

for any $(x, z) \in O_{v}^{\times 2}$, and

$$
\left.\widetilde{\mid g^{(n)}}\left(x, a+\pi^{j} y\right)\right|_{v} ^{s}= \begin{cases}q^{-j s} & \text { if } 1 \leq j \leq 2 n-1 \\ q^{-2 n s}\left|\delta_{1, j}(x, y) y+\delta_{2, j}(x, y) x^{2}\right|_{v}^{s} & \text { if } j=2 n \\ q^{-2 n s} & \text { if } j \geq 2 n+1\end{cases}
$$

We note that the explicit computation of the absolute values $\left.\widetilde{\mid g^{(n)}}\left(x, 1+\pi^{j} y\right)\right|_{v}$ and $\left.\widetilde{g^{(n)}}\left(x, a+\pi^{j} y\right)\right|_{v}$ depends on an explicit description of the sets

$$
\left\{(w, z) \in \mathbb{R}^{2} \mid w \leq \min \{2 z, 2 n\}\right\}
$$

and

$$
\left\{(w, z) \in \mathbb{R}^{2} \mid w \leq \min \{z, 2 n\}\right\},
$$

respectively, for a fixed $n$.

Now, from (3.25), (3.29), (3.30) and the identity $\sum_{k=A}^{B} z^{k}=\frac{z^{A}-z^{B+1}}{1-z}$, we obtain the following explicit expression for $I\left(s, g^{(n)}, v\right)$ :

$$
\begin{aligned}
I\left(s, g^{(n)}, v\right)= & q^{-1}\left(1-q^{-1}\right)(q-3)+\left(1-q^{-1}\right)^{2} \frac{q^{-1-2 s}}{1-q^{-1-2 s}} \\
& -\left(1-q^{-1}\right)^{2} \frac{q^{-n-2 n s}}{1-q^{-1-2 s}}+q^{-n-2 n s} I_{1}(s)+q^{-1}\left(1-q^{-1}\right) q^{-n-2 n s} \\
& +\left(1-q^{-1}\right)^{2} \frac{q^{-1-s}}{1-q^{-1-s}}-\left(1-q^{-1}\right)^{2} \frac{q^{-2 n-2 n s}}{1-q^{-1-s}}+q^{-2 n-2 n s} I_{2}(s) \\
& +q^{-1}\left(1-q^{-1}\right) q^{-2 n-2 n s},
\end{aligned}
$$

where

$$
\begin{aligned}
& I_{1}(s):=\int_{O_{v}^{\times 2}}\left|\gamma_{1, n}(x, y) y^{2}+\gamma_{2, n}(x, y) x^{2}\right|_{v}^{s}|d x d z|, \\
& I_{2}(s):=\int_{O_{v}^{\times 2}}\left|\delta_{1,2 n}(x, y) y+\delta_{2,2 n}(x, y) x^{2}\right|_{v}^{s}|d x d z| .
\end{aligned}
$$

Since the reduction modulo $\pi$ of the polynomials $\gamma_{1, n}(x, y) y^{2}+\gamma_{2, n}(x, y) x^{2}$ and $\delta_{1,2 n}(x, y) y+\delta_{2,2 n}(x, y) x^{2}$ does not have singular points on $\mathbb{F}_{q}^{\times 2}$, because the characteristic of the residue field is different from 2, SPF implies that

$$
\begin{aligned}
& I_{1}(s)=\frac{U_{1}\left(q^{-s}\right)}{1-q^{-1-s}}, \\
& I_{2}(s)=\frac{U_{2}\left(q^{-s}\right)}{1-q^{-1-s}},
\end{aligned}
$$

where $U_{1}\left(q^{-s}\right)$ and $U_{1}\left(q^{-s}\right)$ are polynomials in $q^{-s}$, independent of $j$ and $n$. 
Finally, from (3.19) and (3.31), we obtain the following explicit expression for $Z\left(s, f, v, \Delta_{5}\right)$ :

$$
\begin{aligned}
Z\left(s, f, v, \Delta_{5}\right)= & \frac{(q-3)\left(1-q^{-1}\right) q^{-6-18 s}}{\left(1-q^{-5-18 s}\right)}+\frac{\left(1-q^{-1}\right)^{2} q^{-6-20 s}}{\left(1-q^{-1-2 s}\right)\left(1-q^{-5-18 s}\right)} \\
& -\frac{\left(1-q^{-1}\right)^{2} q^{-6-20 s}}{\left(1-q^{-1-2 s}\right)\left(1-q^{-6-20 s}\right)}+\frac{q^{-6-20 s} U_{1}\left(q^{-s}\right)}{\left(1-q^{-1-s}\right)\left(1-q^{-6-20 s}\right)} \\
& +\frac{\left(1-q^{-1}\right) q^{-7-20 s}}{\left(1-q^{-6-20 s}\right)}+\frac{\left(1-q^{-1}\right)^{2} q^{-6-19 s}}{\left(1-q^{-1-s}\right)\left(1-q^{-5-18 s}\right)} \\
& -\frac{\left(1-q^{-1}\right)^{2} q^{-7-20 s}}{\left(1-q^{-1-s}\right)\left(1-q^{-7-20 s}\right)}+\frac{q^{-7-20 s} U_{2}\left(q^{-s}\right)}{\left(1-q^{-1-s}\right)\left(1-q^{-7-20 s}\right)} \\
& +\frac{\left(1-q^{-1}\right) q^{-8-20 s}}{\left(1-q^{-7-20 s}\right)} .
\end{aligned}
$$

From the explicit formulas for $Z\left(s, f, v, \Delta_{i}\right), i=1,2, \ldots, 8$, and $Z\left(s, f, v, O_{v}^{\times 2}\right)$ (see (3.18), (3.34) $)$, it follows that the real parts of the poles of $Z(s, f, v)$ belong to the set

$$
\{-1\} \cup\left\{-\frac{5}{18},-\frac{1}{3}\right\} \cup\left\{-\frac{1}{2},-\frac{3}{10}\right\} \cup\left\{-\frac{7}{20}\right\}
$$

\section{Arithmetic Newton polygons}

\section{AND ARITHMETIC NON-DEGENERATE POLYNOMIALS}

In this section we introduce the notions of arithmetic Newton polygon and arithmetic non-degeneracy for polynomials in two variables. The set of arithmetically non-degenerate polynomials strictly contains the set of non-degenerate polynomials in the sense of Kouchnirenko. In addition, an explicit list of candidates for the poles of a local zeta function associated to an arithmetically non-degenerate polynomial can be given in terms of the corresponding arithmetic Newton polygon (see Section 6).

\subsection{Arithmetically non-degenerate semi-quasihomogeneous polynomials.}

Definition 4.1. Let $K$ be a field, and $a, b$ two coprime positive integers. A polynomial $g(x, y) \in K[x, y]$ is quasihomogeneous with respect to the weight $(a, b)$, if it has the form

$$
g(x, y)=c x^{u} y^{v} \prod_{i=1}^{l}\left(y^{a}-\alpha_{i} x^{b}\right)^{e_{i}}, c \in K^{\times} .
$$

From the definition it follows that a quasihomogeneous polynomial $g(x, y)$ satisfies

$$
g\left(t^{a} x, t^{b} y\right)=t^{d} g(x, y), \text { for every } t \in K^{\times} .
$$

The integer $d$ is called the weighted degree of $g(x, y)$ with respect to $(a, b)$. We note that our definition of quasihomogeneity coincides with the standard one (i.e. with (4.2) ) after a finite extension of $K$. 
Definition 4.2. A polynomial $g(x, y) \in K[x, y]$ is a semi-quasihomogeneous polynomial with respect to the weight $(a, b)$, if it has the form

$$
g(x, y)=\sum_{j=0}^{l_{g}} g_{j}(x, y),
$$

where each $g_{j}(x, y)$ is quasihomogeneous of weighted degree $d_{j}$ with respect to $(a, b)$, and $d_{0}<d_{1}<\cdots<d_{l_{g}}$.

The polynomial $g_{0}(x, y)$ is called the quasihomogeneous part of $g(x, y)$. If

$$
g_{0}(x, y)=c x^{u_{0}} y^{v_{0}} \prod_{i=1}^{l_{0}}\left(y^{a}-\alpha_{i, 0} x^{b}\right)^{e_{i, 0}}
$$

has singular points on the torus $\left(K^{\times}\right)^{2}$, i.e. if $e_{i, 0}>1$, for some $i=1,2, \ldots, l_{0}$, then $g(x, y)$ is degenerate, in the sense of Kouchnirenko, with respect to its geometric Newton polygon $\Gamma^{\text {geom }}(g)$.

We put

$$
f_{j}(x, y):=c_{j} x^{u_{j}} y^{v_{j}} \prod_{i=1}^{l_{j}}\left(y^{a}-\alpha_{i, j} x^{b}\right)^{e_{i, j}}, c_{j} \in K^{\times},
$$

$d_{j}$ is the weighted degree of $f_{j}(x, y)$ with respect to $(a, b)$, i.e.

$$
d_{j}:=a b\left(\sum_{i=1}^{l_{j}} e_{i, j}\right)+a u_{j}+b v_{j},
$$

and

$$
f(x, y)=\sum_{j=0}^{l_{f}} f_{j}(x, y),
$$

with $d_{0}<d_{1}<\cdots<d_{l_{f}}$. From now on, we shall assume that $f(x, y)$ is a degenerate polynomial in the sense of Kouchnirenko.

Definition 4.3. Let $f(x, y) \in K[x, y]$ be a semi-quasihomogeneous polynomial, $\theta \in K^{\times}$a fixed root of $f_{0}\left(1, \theta^{a}\right)=0$, and $e_{j, \theta}$ the multiplicity of $\theta$ as a root of the polynomial function $f_{j}\left(1, y^{a}\right)$. To each $f_{j}(x, y)$ in (4.5), we associate a straight line of the form

$$
w_{j, \theta}(z):=\left(d_{j}-d_{0}\right)+e_{j, \theta} z, j=0,1, \ldots, l_{f},
$$

and define the arithmetic Newton polygon $\Gamma_{f, \theta}$ of $f(x, y)$ at $\theta$ as

$$
\Gamma_{f, \theta}:=\left\{(z, w) \in \mathbb{R}_{+}^{2} \mid w \leq \min _{0 \leq j \leq l_{f}}\left\{w_{j, \theta}(z)\right\}\right\} .
$$

The arithmetic Newton polygon $\Gamma^{A}(f)$ of $f(x, y)$ is defined to be the set

$$
\Gamma^{A}(f):=\left\{\Gamma_{f, \theta} \mid \theta \in K^{\times}, f_{0}\left(1, \theta^{a}\right)=0\right\} .
$$

We note that $\theta$ is one of the $\alpha_{i, 0}$. In addition, $\Gamma^{A}(f)$ is a finite collection of convex sets, and not just a convex set as $\Gamma^{\text {geom }}(f)$ is.

If the polynomial $f_{0}(x, y)$ is interpreted as the weighted tangent cone of $f(x, y)$, then for each tangent direction of the form $(\theta: 1), \theta \in K^{\times}$, there exists an arithmetic Newton polygon $\Gamma_{f, \theta}$ at $\theta$, and all these Newton polygons constitute the arithmetic Newton polygon $\Gamma^{A}(f)$ of $f(x, y)$. 
Let $\theta$ be a fixed root of $f_{0}\left(1, \theta^{a}\right)=0$. A point $Q$ of the topological boundary of $\Gamma_{f, \theta}$ is called a vertex, if $Q=(0,0)$ or if it is the intersection point of two different straight lines of the form $w_{j, \theta}(z)$. Let $Q_{k}, k=0,1, \ldots, r$, denote the vertices of the topological boundary of $\Gamma_{f, \theta}$, with $Q_{0}:=(0,0)$. The boundary of $\Gamma_{f, \theta}$ is formed by $r$ straight segments, a half-line, and the non-negative part of the horizontal axis of the $(w, z)$-plane. We put

$$
w_{k, \theta}(z)=\left(\mathcal{D}_{k}-d_{0}\right)+\mathcal{E}_{k} z, \quad k=1,2, \ldots, r,
$$

for the equation of the straight segment between $Q_{k-1}$ and $Q_{k}$, and

$$
w_{r+1, \theta}(z)=\left(\mathcal{D}_{r+1}-d_{0}\right)+\mathcal{E}_{r+1} z,
$$

for the equation of the half-line starting at $Q_{r}$. With this notation we have

$$
Q_{k}=\left(\tau_{k},\left(\mathcal{D}_{k}-d_{0}\right)+\mathcal{E}_{k} \tau_{k}\right), k=1,2, \ldots, r,
$$

with

$$
\tau_{k}:=\frac{\left(\mathcal{D}_{k+1}-\mathcal{D}_{k}\right)}{\mathcal{E}_{k}-\mathcal{E}_{k+1}}>0, k=1,2, \ldots, r .
$$

We note that $\mathcal{D}_{k}=d_{j_{k}}$ and $\mathcal{E}_{k}=e_{j_{k}, \theta}$, for some index $j_{k}$. In particular, $\mathcal{D}_{1}=d_{0}$, $\mathcal{E}_{1}=e_{0, \theta}$, and the first equation is $w_{1, \theta}(z)=\mathcal{E}_{1} z$. The slope $\mathcal{E}_{r+1}$ may be 0.

Let $Q$ be a vertex of the boundary of $\Gamma_{f, \theta}$. The face function $f_{Q}(x, y)$ is defined to be the polynomial

$$
f_{Q}(x, y):=\sum_{w_{j, \theta}(Q)=0} f_{j}(x, y),
$$

where $w_{j, \theta}(z)$ is the straight line corresponding to $f_{j}(x, y)$.

Now we introduce a new notion of non-degeneracy with respect to an arithmetic Newton polygon.

Definition 4.4. A semi-quasihomogeneous polynomial $f(x, y) \in K[x, y]$ is called arithmetically non-degenerate with respect to $\Gamma_{f, \theta}$ at $\theta$, if:

(1) the origin of $K^{2}$ is a singular point of $f(x, y)$;

(2) the polynomial $f(x, y)$ does not have singular points on $\left(K^{\times}\right)^{2}$;

(3) the system of equations

$$
f_{Q}(x, y)=\frac{\partial f_{Q}}{\partial x}(x, y)=\frac{\partial f_{Q}}{\partial y}(x, y)=0
$$

does not have solutions on $\left(K^{\times}\right)^{2}$, for any vertex $Q \neq(0,0)$ of the boundary of $\Gamma_{f, \theta}$.

A semi-quasihomogeneous polynomial $f(x, y) \in K[x, y]$ is called arithmetically non-degenerate with respect to $\Gamma^{A}(f)$, if it is arithmetically non-degenerate with respect to $\Gamma_{f, \theta}$, for each $\theta \in K^{\times}$satisfying $f_{0}\left(1, \theta^{a}\right)=0$.

4.2. Arithmetically non-degenerate polynomials. Let $f(x, y) \in K[x, y]$ be a non-constant polynomial, and $\Gamma^{g e o m}(f)$ its geometric Newton polygon. Each facet $\gamma \subset \Gamma^{g e o m}(f)$ has a perpendicular primitive vector $a_{\gamma}=\left(a_{1}(\gamma), a_{2}(\gamma)\right)$ and the corresponding supporting line has an equation of the form $\left\langle a_{\gamma}, x\right\rangle=d(\gamma)$.

Definition 4.5. Let $f(x, y) \in K[x, y]$ be a non-constant polynomial such that $f(x, y)$ is semi-quasihomogeneous with respect to the weight $a_{\gamma}$, in the sense of Definition 4.2 for every facet $\gamma$ of $\Gamma^{g e o m}(f)$. Let $\Gamma^{A}(f, \gamma)$ be the arithmetic Newton 
polygon of $f(x, y)$ regarded as a semi-quasihomogeneous polynomial with respect to the weight $a_{\gamma}$. The arithmetic Newton polygon $\Gamma^{A}(f)$ of $f(x, y)$ is defined to be the set

$$
\Gamma^{A}(f):=\bigcup_{\left\{\gamma \text { a facet of } \Gamma^{g e o m}(f)\right\}} \Gamma^{A}(f, \gamma) .
$$

We note that the construction of the arithmetic polygon of $f$ requires that $f_{\gamma}$ can be factored as in (4.4), for every facet $\gamma$ of $\Gamma^{g e o m}(f)$. This condition can always be satisfied by passing to a finite extension of $K$.

Definition 4.6. A polynomial $f(x, y) \in K[x, y]$ is called arithmetically nondegenerate with respect to its arithmetic Newton polygon, if for every facet $\gamma$ of $\Gamma^{\text {geom }}(f)$, the semi-quasihomogeneous polynomial $f(x, y)$, with respect to the weight $a_{\gamma}$, is arithmetically non-degenerate with respect to $\Gamma^{A}(f, \gamma)$.

We put $K=\mathbb{C}$, and fix a geometric Newton polygon $\Gamma^{\text {geom }} \subset \mathbb{R}^{2}$. Then the set of degenerate polynomials in two variables with respect to $\Gamma^{\text {geom }}$ contains an open set, in the Zariski topology, consisting of arithmetically non-degenerate polynomials. The proof of this fact is similar to [1, p. 157].

4.3. Examples. The following three examples illustrate the above definitions for specific polynomials; we assume that $K$ is a field of characteristic zero.

Example 4.1. We set $f(x, y)=\left(y^{3}-x^{2}\right)^{2}+x^{4} y^{4} \in K[x, y]$. The polynomial $f(x, y)$ is a degenerate, in the sense of Kouchnirenko, semi-quasihomogeneous polynomial with respect to the weight $(3,2)$. The origin of $K^{2}$ is the only singular point of $f(x, y)$. In this case the arithmetic Newton polygon of $f(x, y)$ is equal to $\Gamma_{f, 1}$. The boundary of the Newton polygon $\Gamma_{f, 1}$ is formed by the straight segments

$$
\begin{aligned}
& w_{1,1}(z)=2 z, \quad 0 \leq z \leq 4, \\
& w_{2,1}(z)=8, \quad z \geq 4,
\end{aligned}
$$

and the half-line $\left\{(z, w) \in \mathbb{R}_{+}^{2} \mid w=0\right\}$. Thus $\mathcal{D}_{1}=d_{0}=12, \mathcal{E}_{1}=2, \mathcal{D}_{2}=20$, $\tau_{0}=0, \tau_{1}=4$. The arithmetic Newton polygon $\Gamma_{f, 1}$ is shown in Figure 1 .

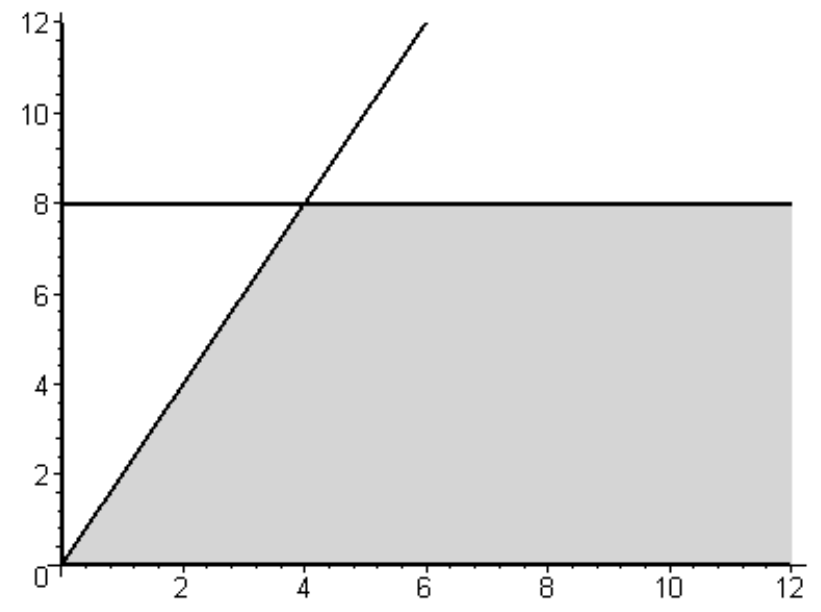

FiguRE 1. $\Gamma^{A}(f)$. 
The face functions are

$$
f_{(0,0)}(x, y)=\left(y^{3}-x^{2}\right)^{2}, f_{(4,8)}(x, y)=\left(y^{3}-x^{2}\right)^{2}+x^{4} y^{4} .
$$

Since $f_{(4,8)}(x, y)$ does not have singular points on $K^{\times 2}, f(x, y)$ is arithmetically non-degenerate.

Example 4.2. We set

$$
g(x, y)=\left(y^{3}-x^{2}\right)^{5}+\left(y^{3}-x^{2}\right)^{3} x^{6} y^{3}+\left(y^{3}-x^{2}\right)^{2} x^{12}+x^{24} \in K[x, y] .
$$

The polynomial $g(x, y)$ is a degenerate, in the sense of Kouchnirenko, semi-quasihomogeneous polynomial with respect to the weight $(3,2)$. The origin of $K^{2}$ is the only singular point of $g(x, y)$. It can be decomposed as

$$
g(x, y)=g_{0}(x, y)+g_{1}(x, y)+g_{2}(x, y)+g_{3}(x, y)
$$

where

$$
\begin{aligned}
& g_{0}(x, y)=\left(y^{3}-x^{2}\right)^{5}, \text { hence } d_{0}=30, e_{0,1}=5 \text { and } w_{0,1}=5 z ; \\
& g_{1}(x, y)=\left(y^{3}-x^{2}\right)^{3} x^{6} y^{3}, \text { hence } d_{1}=42, e_{1,1}=3 \text { and } w_{1,1}=12+3 z ; \\
& g_{2}(x, y)=\left(y^{3}-x^{2}\right)^{2} x^{12}, \text { hence } d_{2}=48, e_{2,1}=2 \text { and } w_{2,1}=18+2 z ; \\
& g_{3}(x, y)=x^{24}, \text { hence } d_{3}=72, e_{3,1}=0 \text { and } w_{3,1}=42 .
\end{aligned}
$$

Since $g_{0}\left(1, \theta^{a}\right)=0$ has only one root, $\theta=1$, the arithmetic Newton polygon $\Gamma^{A}(g)$ of $g(x, y)$ is equal to $\Gamma_{g, 1}$. The boundary of $\Gamma^{A}(g)$ is formed by the straight segments

$$
\begin{aligned}
& w_{1,1}(z)=5 z, \quad 0 \leq z \leq 6, \\
& w_{2,1}(z)=18+2 z, \quad 6 \leq z \leq 12, \\
& w_{3,1}(z)=42, \quad z \geq 12,
\end{aligned}
$$

and the half-line $\left\{(z, w) \in \mathbb{R}_{+}^{2} \mid w=0\right\}$. Thus $\mathcal{D}_{1}=d_{0}=30, \mathcal{E}_{1}=5, \mathcal{D}_{2}=48$, $\mathcal{E}_{2}=2, \mathcal{D}_{3}=72, \mathcal{E}_{3}=0, \tau_{0}=0, \tau_{1}=6, \tau_{2}=12$.

We observe that the line $w(z)=12+3 z$ meets $\Gamma_{g, 1}$ only at $(6,30)$. The arithmetic Newton polygon of $g$ is shown in Figure 2 .

The face functions are

$$
\begin{aligned}
g_{(0,0)}(x, y) & =\left(y^{3}-x^{2}\right)^{5} \\
g_{(6,30)}(x, y) & =\left(y^{3}-x^{2}\right)^{5}+\left(y^{3}-x^{2}\right)^{3} x^{6} y^{3}+\left(y^{3}-x^{2}\right)^{2} x^{12}, \\
g_{(12,40)}(x, y) & =\left(y^{3}-x^{2}\right)^{2} x^{12}+x^{24} .
\end{aligned}
$$

Since $g_{(6,30)}(x, y)=\left(y^{3}-x^{2}\right)^{2}\left(\left(y^{3}-x^{2}\right)^{3}+\left(y^{3}-x^{2}\right)+x^{12}\right)$ has singular points on $\left(K^{\times}\right)^{2}, g(x, y)$ is arithmetically degenerate.

Example 4.3. We set

$$
h(x, y)=\left(y^{5}-x^{3}\right)^{4}\left(y^{5}-a x^{3}\right)\left(y^{5}-b x^{3}\right)+x^{20} \in K[x, y],
$$

with $1 \neq a \neq b$. The polynomial $h(x, y)$ is a degenerate, in the sense of Kouchnirenko, semi-quasihomogeneous polynomial with respect to the weight $(5,3)$. The origin of $K^{2}$ is a singular point of $h(x, y)$, and there are no singularities on the torus $\left(K^{\times}\right)^{2}$. In this case the arithmetic Newton polygon of $h(x, y)$ is equal to 


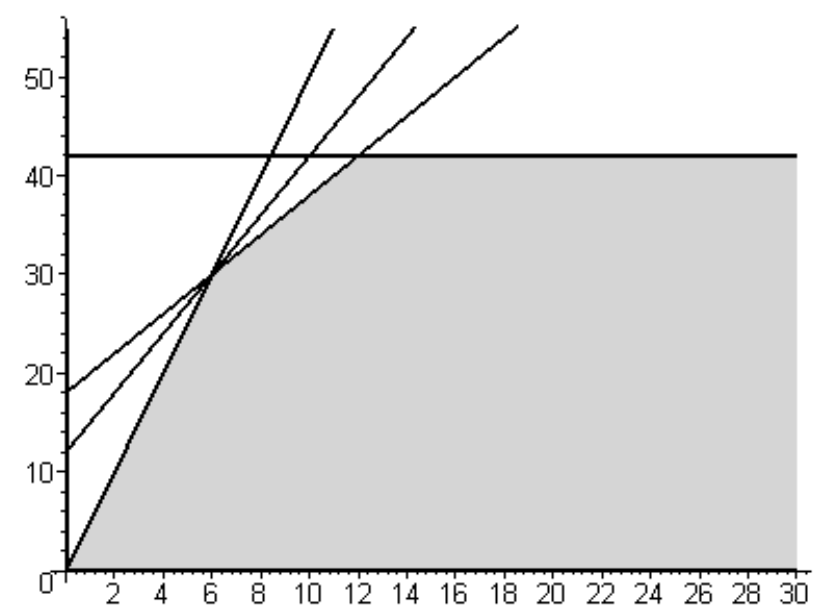

Figure 2. $\Gamma^{A}(g)$.

$\Gamma^{A}(h)=\left\{\Gamma_{h, 1}, \Gamma_{h, a}, \Gamma_{h, b}\right\}$. The boundary of the Newton polygon $\Gamma_{h, 1}$ is formed by the straight segments

$$
\begin{aligned}
& w_{1,1}(z)=4 z, \quad 0 \leq z \leq \frac{5}{2}, \\
& w_{2,1}(z)=10, \quad z \geq \frac{5}{2},
\end{aligned}
$$

and the half-line $\left\{(z, w) \in \mathbb{R}_{+}^{2} \mid w=0\right\}$. The arithmetic Newton polygon $\Gamma_{h, 1}$ is shown in Figure 3.

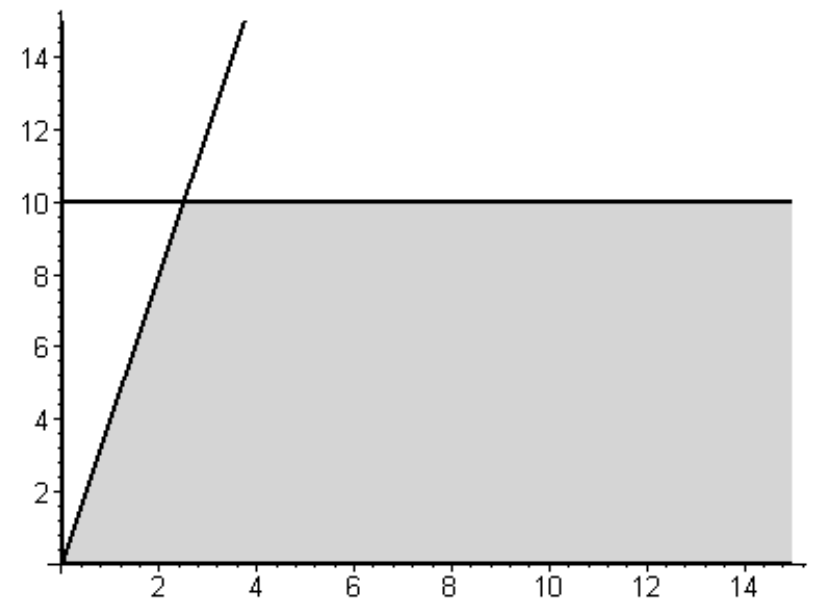

Figure $3 . \Gamma_{h, 1}$.

The face functions are

$$
\begin{aligned}
h_{(0,0)}(x, y) & =\left(y^{5}-x^{3}\right)^{4}\left(y^{5}-a x^{3}\right)\left(y^{5}-b x^{3}\right), \\
h_{\left(\frac{5}{2}, 10\right)}(x, y) & =\left(y^{5}-x^{3}\right)^{4}\left(y^{5}-a x^{3}\right)\left(y^{5}-b x^{3}\right)+x^{20} .
\end{aligned}
$$


Since

$$
h_{\left(\frac{5}{2}, 10\right)}(x, y)=\left(y^{5}-x^{3}\right)^{4}\left(y^{5}-a x^{3}\right)\left(y^{5}-b x^{3}\right)+x^{20}
$$

has no singular points on $\left(K^{\times}\right), f(x, y)$ is arithmetically non-degenerate with respect to $\Gamma_{h, 1}$.

The boundary of the Newton polygon $\Gamma_{h, a}$ is formed by the straight segments

$$
\begin{aligned}
& w_{1, a}(z)=z, \quad 0 \leq z \leq 10, \\
& w_{2, a}(z)=10, \quad z \geq 10,
\end{aligned}
$$

and the half-line $\left\{(z, w) \in \mathbb{R}_{+}^{2} \mid w=0\right\}$. The arithmetic Newton polygon $\Gamma_{h, a}$ is shown in Figure 4.

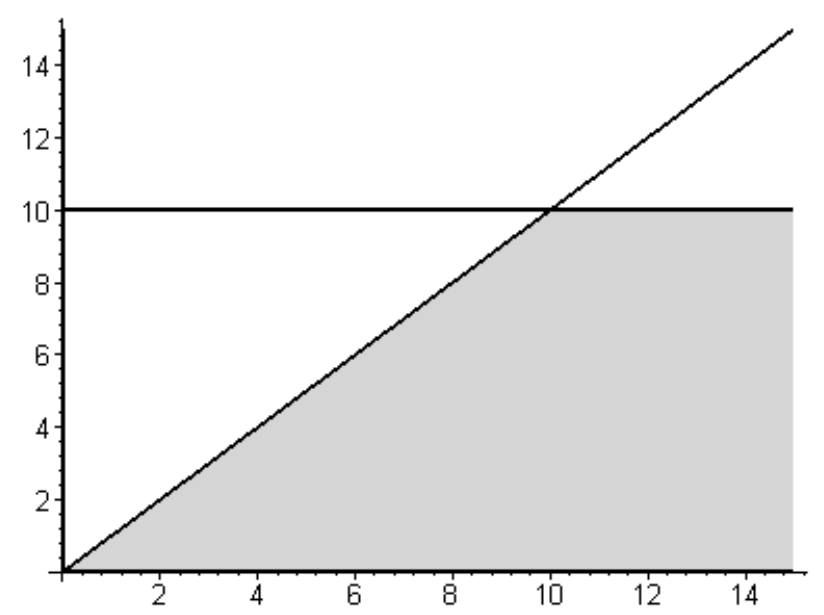

FiguRE 4. $\Gamma_{h, a}$.

The face functions are

$$
\begin{aligned}
h_{(0,0)}(x, y) & =\left(y^{5}-x^{3}\right)^{4}\left(y^{5}-a x^{3}\right)\left(y^{5}-b x^{3}\right), \\
h_{(10,10)}(x, y) & =\left(y^{5}-x^{3}\right)^{4}\left(y^{5}-a x^{3}\right)\left(y^{5}-b x^{3}\right)+x^{20} .
\end{aligned}
$$

Since

$$
h_{(10,10)}(x, y)=\left(y^{5}-x^{3}\right)^{4}\left(y^{5}-a x^{3}\right)\left(y^{5}-b x^{3}\right)+x^{20}
$$

has no singular points on $\left(K^{\times}\right)^{2}, f(x, y)$ is arithmetically non-degenerate with respect to $\Gamma_{h, a}$. The Newton polygon $\Gamma_{h, b}$ is equal to $\Gamma_{h, a}$. Thus the polynomial $h(x, y)$ is arithmetically non-degenerate with respect $\Gamma_{h}^{A}$.

\section{Degenerate integrals of type $Z(s, f, v, \Delta)$}

In this section, using the notion of arithmetic non-degeneracy introduced in the previous section, we explicitly compute degenerate integrals of the type

$$
Z(s, f, v, \Delta)=\int_{E}|f(x, y)|_{v}^{s}|d x d y|,
$$

where

$$
\Delta:=(a, b) \mathbb{R}_{+} \quad \text { and } \quad E:=\left\{(x, y) \in O_{v}^{2} \mid(v(x), v(y)) \in \Delta\right\}
$$


and $f(x, y) \in O_{v}[x, y]$ is a degenerate, in the sense of Kouchnirenko, semi-quasihomogeneous polynomial with respect to the weight $(a, b)$, but arithmetically nondegenerate with respect to $\Gamma^{A}(f)=\bigcup_{\theta} \Gamma_{f, \theta}$. From now on, we shall consider only those $\Gamma_{f, \theta}$ for which $\theta \in O_{v}$. The reason is that the $\Gamma_{f, \theta}$, with $\theta \in L_{v} \backslash O_{v}$, do not contribute to the poles of $Z(s, f, v, \Delta)$.

Definition 5.1. For a semi-quasihomogeneous polynomial $f(x, y) \in L_{v}[x, y]$ nondegenerate with respect to $\Gamma^{A}(f)=\bigcup_{\left\{\theta \in O_{v} \mid f_{0}\left(1, \theta^{a}\right)=0\right\}} \Gamma_{f, \theta}$, we define

$$
\mathcal{P}\left(\Gamma_{f, \theta}\right):=\bigcup_{i=1}^{r_{\theta}}\left\{-\frac{1}{\mathcal{E}_{i}},-\frac{(a+b)+\tau_{i}}{\mathcal{D}_{i+1}+\mathcal{E}_{i+1} \tau_{i}},-\frac{(a+b)+\tau_{i}}{\mathcal{D}_{i}+\mathcal{E}_{i} \tau_{i}}\right\} \cup \bigcup_{\left\{\mathcal{E}_{r+1} \neq o\right\}}\left\{-\frac{1}{\mathcal{E}_{r+1}}\right\}
$$

and

$$
\mathcal{P}\left(\Gamma^{A}(f)\right):=\bigcup_{\left\{\theta \in O_{v} \mid f_{0}\left(1, \theta^{a}\right)=0\right\}} \mathcal{P}\left(\Gamma_{f, \theta}\right) .
$$

The data $\mathcal{D}_{i}, \mathcal{E}_{i}, \tau_{i}$, for each $i$, are obtained from the equations of the straight segments that form the boundary of $\Gamma_{f, \theta}$.

The main result of this section is the following.

Theorem 5.1. Let $f(x, y)=\sum_{j=0}^{l_{f}} f_{j}(x, y) \in O_{v}[x, y]$ be a semi-quasihomogeneous polynomial, with respect to the weight $(a, b)$, with $a, b$ coprime, and $f_{j}(x, y)$ as in (4.4). If $f(x, y)$ is arithmetically non-degenerate with respect to $\Gamma^{A}(f)$, then the real parts of the poles of $Z(s, f, v, \Delta)$ belong to the set

$$
\{-1\} \cup\left\{-\frac{a+b}{d_{0}}\right\} \cup \mathcal{P}\left(\Gamma^{A}(f)\right)
$$

The proof will be given at the end of this section, and it will be accomplished by explicitly computing several $p$-adic integrals. Moreover the proof provides an effective method for computing these integrals.

The following two examples illustrate the theorem.

Example 5.1. We set $f(x, y)=\left(y^{3}-x^{2}\right)^{2}+x^{4} y^{4} \in L_{v}[x, y]$. We suppose that the characteristic of the residue field is different from 2 . The polynomial $f(x, y)$ is an arithmetically non-degenerate, semi-quasihomogeneous polynomial with respect to the weight $(3,2)$ (see Example 4.1). In this case, we have the following data attached to the arithmetic Newton polygon $\Gamma^{A}(f)=\Gamma_{f, 1}: a=3, b=2, \mathcal{D}_{1}=d_{0}=12$, $\tau_{1}=4, \mathcal{E}_{1}=2, \mathcal{D}_{2}=20$. Thus according the above theorem, the real parts of the poles of the integral $Z(s, f, v)$ belong to the set

$$
\{-1\} \cup\left\{-\frac{5}{12}\right\} \cup\left\{-\frac{1}{2},-\frac{9}{20}\right\} .
$$

In Subsection 3.1, we explicitly computed the local zeta function $Z(s, f, v)$ (cf. (3.17)).

Example 5.2. We set $g(x, y)=\left(y^{3}-x^{2}\right)^{2}\left(y^{3}-a x^{2}\right)+x^{4} y^{4} \in L_{v}[x, y]$, with $a \not \equiv 1$ $\bmod \pi$. We assume that the characteristic of the residue field of $L_{v}$ is different from 2. The polynomial $g(x, y)$ is quasihomogeneous with respect to the weight $(3,2)$. The origin of $L_{v}^{2}$ is the only singular point of $g(x, y)$. 
The arithmetic Newton polygon $\Gamma^{A}(g)=\left\{\Gamma_{g, 1}, \Gamma_{g, a}\right\}$. The boundary of the Newton polygon $\Gamma_{g, 1}$ is formed by

$$
\begin{array}{r}
w_{1,1}(z)=2 z, \quad 0 \leq z \leq 1, \\
w_{2,1}(z)=2, \quad z \geq 1, \\
w(z)=0, \quad z \geq 0 .
\end{array}
$$

Thus $\mathcal{D}_{1}=d_{0}=18, \mathcal{E}_{1}=2, \mathcal{D}_{2}=20, \mathcal{E}_{2}=0, \tau_{0}=0, \tau_{1}=1$. The polynomial is arithmetically non-degenerate with respect to $\Gamma_{g, 1}$. According to the previous theorem the contribution of the arithmetic Newton polygon $\Gamma_{g, 1}$ to the set of real parts of $Z(s, g, v)$ is $\left\{-\frac{1}{2},-\frac{3}{10}\right\}$.

The boundary of the Newton polygon $\Gamma_{g, a}$ is formed by

$$
\begin{array}{r}
w_{1,1}(z)=z, \quad 0 \leq z \leq 2, \\
w_{2,1}(z)=2, \quad z \geq 2 . \\
w(z)=0, \quad z \geq 0 .
\end{array}
$$

Thus $\mathcal{D}_{1}=d_{0}=18, \mathcal{E}_{1}=1, \mathcal{D}_{2}=20, \mathcal{E}_{2}=0, \tau_{0}=0, \tau_{1}=2$. The polynomial is arithmetically non-degenerate with respect to $\Gamma_{g, a}$. According to the previous theorem the contribution of the arithmetic Newton polygon $\Gamma_{g, a}$ to the set of real parts of $Z(s, g, v)$ is $\left\{-1,-\frac{7}{20}\right\}$. Then according to the previous theorem the real parts of the poles of $Z(s, g, v)$ belong to the set $\{-1\} \cup\left\{-\frac{5}{18}\right\} \cup\left\{-\frac{1}{2},-\frac{9}{20}\right\} \cup\left\{-\frac{7}{20}\right\}$. In Subsection 3.5 we explicitly computed the local zeta $Z(s, g, v)$ (cf. (3.35)).

\subsection{Some $p$-adic integrals.}

Proposition 5.1. Let $f(x, y) \in O_{v}[x, y]$ be a semi-quasihomogeneous polynomial, with respect to the weight $(a, b)$, with $a, b$ coprime, and

$$
f^{(m)}(x, y):=\pi^{-d_{0} m} f\left(\pi^{a m} x, \pi^{b m} y\right)=\sum_{j=0}^{l_{f}} \pi^{\left(d_{j}-d_{0}\right) m} f_{j}(x, y),
$$

with $f_{j}(x, y)$ as in (4.4), and $m \geq 1$. Then there exists a measure-preserving bijection

$$
\begin{aligned}
\Phi: O_{v}^{\times 2} & \rightarrow O_{v}^{\times 2}, \\
(u, w) & \rightarrow\left(\Phi_{1}(x, y), \Phi_{2}(x, y)\right),
\end{aligned}
$$

such that $f^{(m)} \circ \Phi(x, y)=u^{N_{i}} w^{M_{i}} \widetilde{f^{(m)}}(u, w)$, with $\widetilde{f^{(m)}}(u, w)$ non-vanishing identically on the hypersurface $u w=0$, and

$$
\widetilde{f^{(m)}}(u, w)=\sum_{j=0}^{l_{f}} \pi^{\left(d_{j}-d_{0}\right) m} \widetilde{f}_{j}(u, w),
$$

with

$$
\begin{gathered}
\tilde{f}_{j}(u, w)=c_{j} u^{A_{j}} w^{B_{j}} \prod_{i=1}^{l_{j}}\left(w-\alpha_{i, j}\right)^{e_{i, j}}, \quad \text { or } \\
\tilde{f}_{j}(u, w)=c_{j} u^{L_{j}} w^{F_{j}} \prod_{i=1}^{l_{j}}\left(1-\alpha_{i, j} u\right)^{e_{i, j}} .
\end{gathered}
$$


Proof. We denote by $\Phi_{1}$ the map

$$
\begin{aligned}
\Phi_{1}(u, w): O_{v}^{\times 2} & \rightarrow O_{v}^{\times 2}, \\
(u, w) & \rightarrow(x, y),
\end{aligned}
$$

with $x=u, y=u w$, and by $\Phi_{2}$ the map

$$
\begin{aligned}
\Phi_{2}(u, w): O_{v}^{\times 2} & \rightarrow O_{v}^{\times 2}, \\
(u, w) & \rightarrow(x, y),
\end{aligned}
$$

with $x=u w, y=w$.

Since $\left|\operatorname{det} \Phi_{1}^{\prime}(u, w)\right|_{v}=1$ and $\left|\operatorname{det} \Phi_{2}^{\prime}(u, w)\right|_{v}=1$, the maps $\Phi_{1}, \Phi_{2}$ give a measure-preserving bijection of $O_{v}^{\times 2}$ to itself. We shall show that $\Phi$ is a finite composition of maps of the form $\Phi_{1}$ or $\Phi_{2}$. The proof will be accomplished by induction on $\min \{a, b\}$.

Case $\min \{a, b\}=1$. In this case, it is sufficient to take $\Phi$ as follows:

$$
\Phi=\left\{\begin{array}{lll}
\underbrace{\Phi_{1} \circ \cdots \circ \Phi_{1}}_{b \text {-times }} & \text { if } & a=1, \\
\underbrace{\Phi_{2} \circ \cdots \circ \Phi_{2}}_{a \text {-times }} & \text { if } & b=1 .
\end{array}\right.
$$

Induction hypothesis. Suppose by the induction hypothesis that the proposition is valid for all polynomials $f^{(m)}(x, y)$ of the form (5.3) satisfying $1 \leq \min \{a, b\} \leq$ $k$, with $k \geq 1$.

Case $\min \{a, b\}=k+1, k \geq 2$. Let $f^{(m)}(x, y)$ be a polynomial of the form (5.3) satisfying $\min \{a, b\}=k+1, k \geq 1$, and $a>b$. By applying the Euclidean algorithm to $a, b$, we have that

$$
a=q_{1} b+r_{1}, \quad 0 \leq r_{1}<b,
$$

for some $q_{1}, r_{1} \in \mathbb{N}$. Because $a, b$ are coprime, necessarily $1 \leq r_{1}<b$.

We set $\Psi=\underbrace{\Phi_{2} \circ \cdots \circ \Phi_{2}}_{q_{1} \text {-times }}$, i.e. $x=u w^{q_{1}}, y=w$, and hence

$$
f^{(m)} \circ \Psi(x, y)=u^{A_{i}} w^{B_{i}} f^{*(m)}(u, w)
$$

with $f^{*(m)}(u, w)$ non-vanishing identically on $u w=0$, and

$$
f^{*(m)}(u, w)=\sum_{j=0}^{l_{f}} \pi^{\left(d_{j}-d_{0}\right) m} f_{j}^{*}(u, w),
$$

with

$$
f_{j}^{*}(u, w)=c_{j} u^{C_{j}} w^{D_{j}} \prod_{i=1}^{l_{j}}\left(w^{r_{1}}-\alpha_{i, j} u^{b}\right)^{e_{i, j}} .
$$

Since $\min \left\{r_{1}, b\right\}=r_{1}$ and $1 \leq r_{1} \leq b-1=k$, by applying the induction hypothesis to the polynomial $f^{*(m)}(u, v)$ in (5.6), there exists a map $\Theta$ that is a finite composition of maps of the form $\Phi_{1}$ or $\Phi_{2}$, such that $\left(f^{(m)} \circ \Psi\right) \circ \Theta=$ $f^{(m)} \circ(\Psi \circ \Theta)=u^{N_{i}} w^{M_{i}} \widetilde{f^{(m)}}(u, v)$, and $\widetilde{f^{(m)}}(u, v)$ has all the properties announced in the proposition. Therefore, it is sufficient to take $\Phi=\Psi \circ \Theta$.

The case $b>a$ is proved in an analogous way. 
Remark 5.1. We can assume without loss of generality that

$$
\widetilde{f^{(m)}}(u, w)=\sum_{j=0}^{l_{f}} \pi^{\left(d_{j}-d_{0}\right) m} \widetilde{f}_{j}(u, w),
$$

with

$$
\tilde{f}_{j}(u, w)=c_{j} u^{A_{j}} w^{B_{j}} \prod_{i=1}^{l_{j}}\left(w-\alpha_{i, j}\right)^{e_{i, j}}, \quad c_{j}, \alpha_{i, j} \in L_{v}^{\times} .
$$

We define

$$
I\left(s, f^{(m)}, v, O_{v}^{\times 2}\right):=\int_{O_{v}^{\times 2}}\left|f^{(m)}(x, y)\right|_{v}^{s}|d x d y| .
$$

Proposition 5.2. Let $f(x, y) \in O_{v}[x, y]$ be a semi-quasihomogeneous polynomial, with respect to the weight $(a, b)$, with $a, b$ coprime, and

$$
f^{(m)}(x, y)=\pi^{-d_{0} m} f\left(\pi^{a m} x, \pi^{b m} y\right)=\sum_{j=0}^{l_{f}} \pi^{\left(d_{j}-d_{0}\right) m} f_{j}(x, y), \quad m \geq 1,
$$

with $f_{j}(x, y)$ as in (4.4). There exists a constant $M_{0}$ such that if $m \geq M_{0}$, then

$$
\begin{gathered}
I\left(s, f^{(m)}, v, O_{v}^{\times 2}\right)=U_{0}\left(q^{-s}\right) \\
+\sum_{\left\{\theta \in O_{v} \mid f_{0}\left(1, \theta^{a}\right)=0\right\}} \sum_{k=1}^{\infty} q^{-k} \int_{O_{v}^{\times} \times O_{v}^{\times}}\left|\widetilde{f^{(m)}}\left(x, \theta+\pi^{k} y\right)\right|_{v}^{s}|d x d y|,
\end{gathered}
$$

where $U_{0}\left(q^{-s}\right)$ is a polynomial with rational coefficients. Moreover, the polynomial $U_{0}\left(q^{-s}\right)$ and the constant $M_{0}$ can be computed effectively.

Proof. Proposition 5.1 implies that

$$
I\left(s, f^{(m)}, v, O_{v}^{\times 2}\right)=\int_{O_{v}^{\times 2}}\left|f^{(m)}(x, y)\right|_{v}^{s}|d x d y|=\int_{O_{v}^{\times 2}}\left|\widetilde{f^{(m)}}(x, y)\right|_{v}^{s}|d x d y| .
$$

We set

$$
\begin{gathered}
R\left(f_{0}\right):=\left\{\theta \in O_{v} \mid f_{0}\left(1, \theta^{a}\right)=0\right\}, \\
l\left(f_{0}\right):=\max _{\substack{\theta \neq \theta^{\prime} \\
\theta, \theta^{\prime} \in R\left(f_{0}\right)}}\left\{v\left(\theta-\theta^{\prime}\right)\right\},
\end{gathered}
$$

and

$$
B(\theta)=B\left(l\left(f_{0}\right), \theta\right):=O_{v}^{\times} \times\left(\theta+\pi^{1+l\left(f_{0}\right)} O_{v}\right), \text { for } \theta \in O_{v} \text {, with } v(\theta) \leq l\left(f_{0}\right) .
$$

With the above notation, if $\theta=\alpha_{i_{0}, 0}$, then

$$
v\left(\tilde{f}_{j}(x, y)\right)= \begin{cases}v\left(c_{j}\right)+B_{j} v(\theta)+\sum_{i=1}^{l_{j}} e_{i, j} v\left(\theta-\alpha_{i, j}\right) & \text { if } f_{j}(1, \theta) \neq 0, \\ v\left(c_{j}\right)+B_{j} v(\theta)+\sum_{\substack{i=1 \\ l_{j}}} e_{i, j} v\left(\theta-\alpha_{i, j}\right) & \\ \quad+e_{i_{0}, j}\left(1+l\left(f_{0}\right)\right)+e_{i_{0}, j} v\left(\frac{y-\theta}{\left.\pi^{1+l\left(f_{0}\right)}\right)}\right) & \text { if } f_{j}(1, \theta)=0,\end{cases}
$$


for every $(x, y) \in B\left(l\left(f_{0}\right), \theta\right)$. We put

$$
\operatorname{Const}(j, \theta):= \begin{cases}v\left(c_{j}\right)+B_{j} v(\theta)+\sum_{i=1}^{l_{j}} e_{i, j} v\left(\theta-\alpha_{i, j}\right) & \text { if } f_{j}(1, \theta) \neq 0, \\ v\left(c_{j}\right)+B_{j} v(\theta)+\sum_{\substack{i=1 \\ i \neq i_{0}}}^{l_{j}} e_{i, j} v\left(\theta-\alpha_{i, j}\right) & \\ +e_{i_{0}, j}\left(1+l\left(f_{0}\right)\right) & \text { if } f_{j}(1, \theta)=0 .\end{cases}
$$

Then, if $\theta \notin R\left(f_{0}\right)$, and $m \geq M_{0}$, with

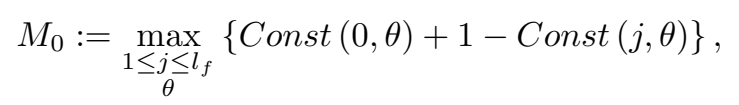

it follows that

$$
v\left(\widetilde{f^{(m)}}(x, y)\right)=\operatorname{Const}(0, \theta),
$$

for every $(x, y) \in B\left(l\left(f_{0}\right), \theta\right)$. From the above calculations we see that

$$
\int_{B\left(l\left(f_{0}\right), \theta\right)}\left|\widetilde{f^{(m)}}(x, y)\right|_{v}^{s}|d x d y|=\mid \text { Const }\left.(0, \theta)\right|_{v} ^{s} \text {, if } \theta \notin R\left(f_{0}\right) \text {, and } m \geq M_{0} \text {. }
$$

By subdividing $O_{v}^{\times} \times O_{v}^{\times}$into equivalence classes modulo $\pi^{1+l\left(f_{0}\right)}$, and using (5.11) and (5.12), we have that

$$
I\left(s, f^{(m)}, v, O_{v}^{\times 2}\right)=U_{0}\left(q^{-s}\right)+\sum_{\theta \in R\left(f_{0}\right)_{B}} \int_{B(\theta)}\left|\widetilde{f^{(m)}}(x, y)\right|_{v}^{s}|d x d y|,
$$

for $m \geq M_{0}$, where $U_{0}\left(q^{-s}\right)$ is a polynomial with rational coefficients. Moreover, the polynomial $U_{0}\left(q^{-s}\right)$ and the constant $M_{0}$ can be computed effectively.

The integral $\int_{B(\theta)}\left|\widetilde{f^{(m)}}(x, y)\right|_{v}^{s}|d x d y|$, for $\theta \in R\left(f_{0}\right)$, admits the following expansion:

$$
\begin{aligned}
& \sum_{k=1}^{\infty} q^{-k} \int_{O_{v}^{\times} \times O_{v}^{\times}}\left|\widetilde{f^{(m)}}\left(x, \theta+\pi^{k} y\right)\right|_{v}^{s}|d x d y| \\
& =\sum_{k=1}^{1+l\left(f_{0}\right)} q^{-k} \int_{O_{v}^{\times} \times O_{v}^{\times}}\left|\widetilde{f^{(m)}}\left(x, \theta+\pi^{k} y\right)\right|_{v}^{s}|d x d y| \\
& +\sum_{k=2+l\left(f_{0}\right)}^{\infty} q^{-k} \int_{O_{v}^{\times} \times O_{v}^{\times}}\left|\widetilde{f^{(m)}}\left(x, \theta+\pi^{k} y\right)\right|_{v}^{s}|d x d y| \\
& =\frac{U_{1}\left(q^{-s}\right)}{1-q^{-1-s}}+\sum_{k=2+l\left(f_{0}\right)}^{\infty} q^{-k} \int_{O_{v}^{\times} \times O_{v}^{\times}}\left|\widetilde{f^{(m)}}\left(x, \theta+\pi^{k} y\right)\right|_{v}^{s}|d x d y|
\end{aligned}
$$

(cf. Lemma 2.1). We set

$$
J(s, m, \theta):=\sum_{k=2+l\left(f_{0}\right)}^{\infty} q^{-k} \int_{O_{v}^{\times} \times O_{v}^{\times}}\left|\widetilde{f^{(m)}}\left(x, \theta+\pi^{k} y\right)\right|_{v}^{s}|d x d y| .
$$


Then from (5.13) and (5.14) it follows that

$$
I\left(s, f^{(m)}, v, O_{v}^{\times 2}\right)=\frac{U\left(q^{-s}\right)}{1-q^{-1-s}}+\sum_{\theta \in R\left(f_{0}\right)} J(s, m, \theta), \quad m \geq M_{0},
$$

where $U\left(q^{-s}\right)$ is a polynomial with rational coefficients.

The polynomial $\widetilde{f^{(m)}}\left(x, \theta+\pi^{k} y\right)$ can expressed as

$$
\widetilde{f^{(m)}}\left(x, \theta+\pi^{k} y\right)=\sum_{j=0}^{l_{f}} C(j, \theta) \pi^{\left(d_{j}-d_{0}\right) m+k e_{j, \theta}} \gamma_{j}(x, y) y^{e_{j, \theta}},
$$

for $k \geq 2+l\left(f_{0}\right)$, with $C(j, \theta)=\operatorname{Const}(j, \theta) c_{j} \in L_{v}^{\times}$, and $\left|\gamma_{j}(x, y)\right|_{v}=1$, for every $(x, y) \in O_{v}^{\times} \times O_{v}^{\times}$.

Given a real number $x,[x]$ denotes the greatest integer less than or equal to $x$.

Proposition 5.3. With the hypothesis of Proposition 5.2, if $f(x, y)$ is arithmetically non-degenerate with respect to $\Gamma_{f, \theta}$, then

$$
\begin{aligned}
J(s, m, \theta)= & \left(1-q^{-1}\right)^{2} \sum_{i=0}^{r-1} \frac{q^{-\left(1+s \mathcal{E}_{i+1}\right)}}{1-q^{-\left(1+s \mathcal{E}_{i+1}\right)}} q^{-\left(\mathcal{D}_{i+1}-d_{0}\right) m s-\left(1+s \mathcal{E}_{i+1}\right)\left[m \tau_{i}\right]} \\
& -\left(1-q^{-1}\right)^{2} \sum_{i=0}^{r-1} \frac{1}{1-q^{-\left(1+s \mathcal{E}_{i+1}\right)}} q^{-\left(\mathcal{D}_{i+1}-d_{0}\right) m s-\left(1+s \mathcal{E}_{i+1}\right)\left[m \tau_{i+1}\right]} \\
& +\left(1-q^{-1}\right)^{2} \frac{q^{-\left(1+s \mathcal{E}_{r+1}\right)}}{1-q^{-\left(1+s \mathcal{E}_{r+1}\right)}} q^{-\left(\mathcal{D}_{r+1}-d_{0}\right) m s-\left(1+s \mathcal{E}_{r+1}\right)\left[m \tau_{r}\right]} \\
& +\sum_{i=1}^{r} q^{-\left(\mathcal{D}_{i}-d_{0}\right) m s-\left(1+s \mathcal{E}_{i}\right)\left[m \tau_{i}\right]} I_{i}(s),
\end{aligned}
$$

where $\tau_{i}, i=0,1, \ldots, r$, are the abscissas of the vertices of $\Gamma_{f, \alpha_{j, 0}}$, and $I_{i}(s)=$ $\frac{M_{i}\left(q^{-s}, m\right)}{1-q^{-1-s}}, M_{i}\left(q^{-s}, m\right) \in \mathbb{Q}\left[q^{-s}\right], i=1,2, \ldots, r$. Moreover, there exists a constant $c(f)$ such that if $m \geq c(f)$, then $I_{i}(s)$ does not depend on $m$.

Proof. The proof is based on an explicit computation of the $v$-adic absolute value of $\widetilde{f^{(m)}}\left(x, \theta+\pi^{k} y\right)$, when $x, y \in O_{v}^{\times}$. In order to accomplish it, we attach to $\widetilde{f^{(m)}}\left(x, \theta+\pi^{k} y\right)$ the convex set $\Gamma_{f^{(m)}\left(x, \theta+\pi^{k} y\right)}$ defined as follows. We associate to each term

$$
C(j, \theta) \pi^{\left(d_{j}-d_{0}\right) m+k e_{j, \theta}} \gamma_{j}(x, y) y^{e_{j, \theta}}
$$

of $\widetilde{f^{(m)}}\left(x, \theta+\pi^{k} y\right)$ (see (5.16) ) a straight line of the form

$$
\widetilde{w}_{j, \theta}(\widetilde{z}):=\left(d_{j}-d_{0}\right) m+e_{j, \theta} \widetilde{z}, \quad j=0,1, \ldots, l_{f},
$$

and associate to $\widetilde{f^{(m)}}\left(x, \theta+\pi^{k} y\right)$ the convex set

$$
\Gamma_{\widetilde{f^{(m)}\left(x, \theta+\pi^{k} y\right)}}=\left\{(\widetilde{z}, \widetilde{w}) \in \mathbb{R}_{+}^{2} \mid \widetilde{w} \leq \min _{0 \leq j \leq l_{f}}\left\{\widetilde{w}_{j, \theta}(\widetilde{z})\right\}\right\} .
$$

Now, we set

$$
\begin{aligned}
& \Omega_{m}: \quad \mathbb{R}^{2} \longrightarrow \mathbb{R}^{2} \\
& (u, w) \longrightarrow(\widetilde{u}, \widetilde{w}),
\end{aligned}
$$


with $u=\frac{\widetilde{u}}{m}, z=\frac{\widetilde{u}}{m}$. Then $\Omega_{m}\left(\Gamma_{f, \theta}\right)=\Gamma_{\widetilde{f^{(m)}\left(x, \theta+\pi^{k} y\right)}}$ (see Definition 4.3). The homomorphism $\Omega_{m}$ sends the topological boundary of $\Gamma_{f, \theta}$ into the topological boundary of $\Gamma_{\widetilde{f^{(m)}\left(x, \theta+\pi^{j} y\right)}}$. Thus the vertices of $\Gamma_{\widetilde{f^{(m)}\left(x, \theta+\pi^{k} y\right)}}$ are

$$
\Omega_{m}\left(Q_{i}\right):= \begin{cases}(0,0) & \text { if } i=0, \\ \left(m \tau_{i},\left(\mathcal{D}_{i}-d_{0}\right) m+m \mathcal{E}_{i} \tau_{i}\right) & \text { if } i=1,2, \ldots, r\end{cases}
$$

where the $\tau_{i}$ are the abscissas of the vertices of $\Gamma_{f^{(m), \theta}}(\mathrm{cf}$. (4.8), (4.9)).

If

$$
m \geq \max _{j, \theta}\{v(C(j, \theta))\},
$$

then $\left|\widetilde{f^{(m)}}\left(x, \theta+\pi^{k} y\right)\right|_{v}$ can be computed from $\Gamma_{\widetilde{f^{(m)}}\left(x, \theta+\pi^{k} y\right)}$ as follows (we use the notation introduced in (4.6), (4.8), and (4.9)):

If $m \tau_{i}<k<m \tau_{i+1}, i=0,1, \ldots, r-1$, then

$$
\left|\widetilde{f^{(m)}}\left(x, \theta+\pi^{k} y\right)\right|_{v}=q^{-\left(\mathcal{D}_{i+1}-d_{0}\right) m-\mathcal{E}_{i+1} k}|C(i+1, \theta)|_{v},(x, y) \in O_{v}^{\times} \times O_{v}^{\times} .
$$

If $k>m \tau_{r}$, then

$$
\left|\widetilde{f^{(m)}}\left(x, \theta+\pi^{k} y\right)\right|_{v}=q^{-\left(\mathcal{D}_{r+1}-d_{0}\right) m-\mathcal{E}_{r+1} k}|C(r+1, \theta)|_{v},(x, y) \in O_{v}^{\times} \times O_{v}^{\times} .
$$

If $k=m \tau_{i}, i=1,2, \ldots, r$, then

$$
\begin{gathered}
\mid \widetilde{\left.f^{(m)}\left(x, \theta+\pi^{k} y\right)\right|_{v}} \\
=q^{-\left(\mathcal{D}_{i}-d_{0}\right) m-\mathcal{E}_{i} k}\left|\widetilde{f_{\Omega_{m}\left(Q_{i}\right)}^{(m)}}(x, y)+\pi^{m\left(\mathcal{D}_{i+1}-\mathcal{D}_{i}\right)}(\cdots)\right|_{v},(x, y) \in O_{v}^{\times} \times O_{v}^{\times},
\end{gathered}
$$

with

$$
\begin{aligned}
& \widetilde{f_{\Omega_{m}\left(Q_{i}\right)}^{(m)}}(\widetilde{x}, y)+\pi^{m\left(\mathcal{D}_{i+1}-\mathcal{D}_{i}\right)}(\cdots) \\
& \quad=\widetilde{f_{\Omega_{m}\left(Q_{i}\right)}^{(m)}}(x, y)+\pi^{m\left(\mathcal{D}_{i+1}-\mathcal{D}_{i}\right)}\left(\text { terms with weighted degree } \geq \mathcal{D}_{i+1}\right),
\end{aligned}
$$

and

$$
\widetilde{f_{\Omega_{m}\left(Q_{i}\right)}^{(m)}}(x, y)=\sum_{\widetilde{w}_{i, \theta}\left(\Omega_{m}\left(Q_{i}\right)\right)=0} \gamma_{i}(x, y) y^{e_{i, \theta}} ;
$$

here $\widetilde{w}_{i, \theta}(\widetilde{z})$ is the straight line corresponding to the term

$$
\pi^{\left(d_{i}-d_{0}\right) m+k e_{i, \theta}} \gamma_{i}(x, y) y^{e_{i, \theta}} .
$$

In addition, we note that any $k \in N, k \geq 1$, satisfies only one of the following conditions:

$$
\begin{gathered}
{\left[m \tau_{i}\right]+1 \leq k \leq\left[m \tau_{i+1}\right]-1, \quad i=0,1, \ldots, r-1} \\
k=\left[m \tau_{i}\right], \quad i=0,1, \ldots, r
\end{gathered}
$$

or

$$
k \geq\left[m \tau_{r}\right]+1 .
$$


Then by using (5.21), (5.22), (15.23), and the previous observation, it follows that

$$
\begin{aligned}
J(s, m, \theta)= & \sum_{k=2+l\left(f_{0}\right)}^{\infty} q^{-k} \int_{O_{v}^{\times} \times O_{v}^{\times}}\left|\widetilde{f(m)}\left(x, \theta+\pi^{k} y\right)\right|_{v}^{s}|d x d y| \\
= & \left(1-q^{-1}\right)^{2} \sum_{i=0}^{r-1}\left(q^{-\left(\mathcal{D}_{i+1}-d_{0}\right) m s} \sum_{j=\left[m \tau_{i}\right]+1}^{\left[m \tau_{i+1}\right]-1} q^{-\left(1+s \mathcal{E}_{i+1}\right) j}\right) \\
& +\left(1-q^{-1}\right)^{2} \sum_{j=\left[m \tau_{r}\right]+1}^{\infty} q^{-\left(\mathcal{D}_{r+1}-d_{0}\right) m s-\left(1+s \mathcal{E}_{r+1}\right) j} \\
& +\sum_{i=1}^{r} q^{-\left(\mathcal{D}_{i}-d_{0}\right) m s-\left(1+s \mathcal{E}_{i}\right)\left[m \tau_{i}\right]} I_{i}(s)
\end{aligned}
$$

with

$$
I_{i}(s):=\int_{O_{v}^{\times 2}} \mid \widetilde{f_{\Omega_{m}\left(Q_{i}\right)}^{(m)}}(x, y)+\pi^{m\left(\mathcal{D}_{i+1}-\mathcal{D}_{i}\right)} \text { (higher order terms) }\left.\right|_{v}|d x d y|
$$

Since $\widetilde{f_{\Omega_{m}\left(Q_{i}\right)}^{(m)}}(x, y)+\pi^{\left(\mathcal{D}_{i+1}-\mathcal{D}_{i}\right)}$ (higher order terms), and $\widetilde{f_{\Omega_{m}\left(Q_{i}\right)}^{(m)}}(x, y)$ do not have singular points on $\left(L_{v}^{\times}\right)^{2}$ (cf. Proposition 5.1), there exists a constant $c_{0}(f)$ such that

$$
I_{i}(s)=\int_{O_{v}^{\times 2}}\left|\widetilde{f_{\Omega_{m}\left(Q_{i}\right)}^{(m)}}(x, y)\right|_{v}|d x d y|=\frac{U_{i}\left(q^{-s}\right)}{1-q^{-1-s}}, \quad U_{i}\left(q^{-s}\right) \in \mathbb{Q}\left[q^{-s}\right], i=1, \ldots, r,
$$

for $m \geq c_{0}(f)$ (cf. Lemma 2.1). Finally, the result follows from (15.24), by using the algebraic identity

$$
\sum_{k=A}^{B} z^{k}=\frac{z^{A}-z^{B+1}}{1-z}
$$

The constant $c(f)=\max \left\{c_{0}(f), \max _{j, \theta}\{v(C(j, \theta))\}\right\}$.

5.2. Some algebraic identities. The following algebraic identities easily follow from (5.26). These identities will be used later for the explicit computation of certain $p$-adic integrals. Let $s$ be a complex number, and $i, c_{0}$ non-negative integers, with $c_{0} \geq 1$.

$$
\begin{aligned}
S_{1}\left(s, i, c_{0}\right) & :=\sum_{m=c_{0}}^{\infty} q^{-(a+b) m-d_{0} m s-\left(\mathcal{D}_{i+1}-d_{0}\right) m s-\left(1+s \mathcal{E}_{i+1}\right)\left[m \tau_{i}\right]} \\
& =\left\{\begin{array}{clc}
\frac{q^{-c_{0}(a+b)-c_{0} d_{0} s}}{1-q^{-(a+b)-d_{0} s}} & \text { if } & i=0, \\
\sum_{l=0}^{\mathcal{E}_{i}-\mathcal{E}_{i+1}-1}\left(\frac{q^{-B_{i, l}-A_{l}\left(\gamma_{i}+\delta_{i} s\right)}}{1-q^{-\gamma_{i}-\delta_{i} s}}\right) & \text { if } & i=1, \ldots, r,
\end{array}\right.
\end{aligned}
$$


with

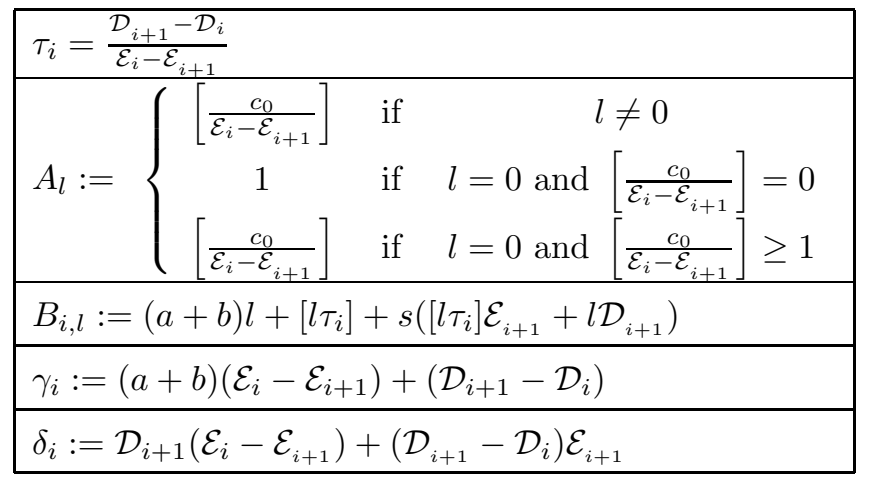

$$
\begin{aligned}
& S_{2}\left(s, i, c_{0}\right):=\sum_{m=1}^{\infty} q^{-(a+b) m-d_{0} m s-\left(\mathcal{D}_{i+1}-d_{0}\right) m s-\left(1+s \mathcal{E}_{i+1}\right)\left[m \tau_{i+1}\right]} \\
& =\sum_{l=0}^{\mathcal{E}_{i+1}-\mathcal{E}_{i+2}-1}\left(\frac{q^{-G_{i, l}-H_{l}\left(\rho_{i}+\sigma_{i} s\right)}}{1-q^{-\rho_{i}-\sigma_{i} s}}\right), \quad i=0, \ldots, r-1,
\end{aligned}
$$

with

\begin{tabular}{|l}
$\tau_{i+1}=\frac{\mathcal{D}_{i+2}-\mathcal{D}_{i+1}}{\mathcal{E}_{i+1}-\mathcal{E}_{i+2}}:=\left\{\begin{array}{ccc|}{\left[\frac{c_{0}}{\mathcal{E}_{i+1}-\mathcal{E}_{i+2}}\right]} & \text { if } & l \neq 0 \\
1 & \text { if } & l=0 \text { and }\left[\frac{c_{0}}{\mathcal{E}_{i+1}-\mathcal{E}_{i+2}}\right]=0 \\
{\left[\frac{c_{0}}{\mathcal{E}_{i+1}-\mathcal{E}_{i+2}}\right]} & \text { if } & l=0 \text { and }\left[\frac{c_{0}}{\mathcal{E}_{i+1}-\mathcal{E}_{i+2}}\right] \geq 1 \\
\hline G_{i, l}:=(a+b) l+\left[l \tau_{i+1}\right]+s\left(\left[l \tau_{i+1}\right] \mathcal{E}_{i+1}+l \mathcal{D}_{i+1}\right)\end{array}\right.$ \\
\hline$\rho_{i}:=(a+b)\left(\mathcal{E}_{i+1}-\mathcal{E}_{i+2}\right)+\left(\mathcal{D}_{i+2}-\mathcal{D}_{i+1}\right)$ \\
\hline$\delta_{i}:=\mathcal{D}_{i+1}\left(\mathcal{E}_{i+1}-\mathcal{E}_{i+2}\right)+\left(\mathcal{D}_{i+2}-\mathcal{D}_{i+1}\right) \mathcal{E}_{i+1}$ \\
\hline
\end{tabular}

Remark 5.2. With the above notation, the real parts of the poles of $S_{1}\left(s, i, c_{0}\right)$, $S_{2}\left(s, i, c_{0}\right)$ belong to the set $P\left(\Gamma_{f, \theta}\right) \cup\left\{-\frac{a+b}{d_{0}}\right\}$.

5.3. Proof of Theorem 5.1. The integral $Z(s, f, v, \Delta)$ admits the following expansion:

$$
\begin{aligned}
Z(s, f, v, \Delta) & =\sum_{m=1}^{\infty} \int_{\pi^{a m} O_{v}^{\times} \times \pi^{b m} O_{v}^{\times}}|f(x, y)|_{v}^{s}|d x d y| \\
& =\sum_{m=1}^{\infty} q^{-(a+b) m-d_{0}} m s \int_{O_{\Upsilon_{v}}}\left|f^{(m)}(x, y)\right|_{v}^{s}|d x d y|,
\end{aligned}
$$

with

$$
f^{(m)}(x, y)=\pi^{-m d_{0}} f\left(\pi^{a m} x, \pi^{b m} y\right)=\sum_{j=0}^{l_{f}} \pi^{\left(d_{j}-d_{0}\right) m} f_{j}(x, y) .
$$


The integral

$$
I\left(f^{(m)}, s, v\right)=\int_{O_{v}^{\times 2}}\left|f^{(m)}(x, y)\right|_{v}^{s}|d x d y|
$$

is equal to

$$
I\left(s, f^{(m)}, v, O_{v}^{\times 2}\right)=\frac{U_{1}\left(q^{-s}\right)}{1-q^{-1-s}}+\sum_{\left\{\theta \in O_{v}^{\times} \mid f_{0}\left(1, \theta^{a}\right)=0\right\}} J(s, m, \theta),
$$

for $m$ big enough (cf. 5.15). Thus from (5.27) and (5.28), it follows that

$$
\begin{aligned}
Z(s, f, v, \Delta)= & \frac{U\left(q^{-s}\right)}{1-q^{-1-s}} \\
& +\sum_{\left\{\theta \in O_{v}^{\times} \mid f_{0}\left(1, \theta^{a}\right)=0\right\}}\left(\sum_{m=c(f)}^{\infty} q^{-(a+b) m-d_{0} m s} J(s, m, \theta)\right),
\end{aligned}
$$

where the constant $c(f)$ is defined in Proposition 5.3 By using the explicit formula for $J(s, m, \theta)$ given in Proposition 5.3 for $m \geq c(f)$, and the algebraic identities given in Subsection 5.2, we have that

$$
\begin{aligned}
\sum_{m=c(f)}^{\infty} q^{-(a+b) m-d_{0} m s} J(s, m, \theta)= & \frac{U_{\theta}\left(q^{-s}\right)}{\left(1-q^{-1-s}\right)} \\
& +\left(1-q^{-1}\right)^{2} \sum_{i=0}^{r-1} \frac{q^{-\left(s \mathcal{E}_{i+1}+1\right)}}{1-q^{-\left(s \mathcal{E}_{i+1}+1\right)}} S_{1}\left(s, i, c_{0}\right) \\
& -\left(1-q^{-1}\right)^{2} \sum_{i=0}^{r-1} \frac{1}{1-q^{-\left(1+s \mathcal{E}_{i+1}\right)}} S_{2}\left(s, i, c_{0}\right) \\
& +\left(1-q^{-1}\right)^{2} \frac{q^{-\left(1+s \mathcal{E}_{r+1}\right)}}{1-q^{-\left(1+s \mathcal{E}_{r+1}\right)}} S_{1}\left(s, r, c_{0}\right) \\
& +\sum_{i=1}^{r} I_{i}(s) S_{2}\left(s, i-1, c_{0}\right),
\end{aligned}
$$

where $U_{\theta}\left(q^{-s}\right) \in \mathbb{Q}\left[q^{-s}\right]$, and the data $\mathcal{E}_{i}, \mathcal{D}_{i}, i=1,2, \ldots, r$, depend on the arithmetic Newton polygon $\Gamma_{f, \theta}$. Now by Remark 5.2 the real parts of the poles of $S_{1}\left(s, i, c_{0}\right), S_{2}\left(s, i, c_{0}\right)$ belong to the set $\mathcal{P}\left(\Gamma_{f, \theta}\right)$. Then the real parts of the poles of

$$
\sum_{\left\{\theta \in O_{v}^{\times} \mid f_{0}\left(1, \theta^{a}\right)=0\right\}}\left(\sum_{m=c(f)}^{\infty} q^{-(a+b) m-d_{0} m s} J(s, m, \theta)\right)
$$

belong to the set $\left\{-\frac{a+b}{d_{0}}\right\} \cup \bigcup_{\left\{\theta \in O_{v} \mid f_{0}\left(1, \theta^{a}\right)=0\right\}} \mathcal{P}\left(\Gamma_{f, \theta}\right)$, and from (5.29) it follows that the real parts of the poles of $Z(s, f, v, \Delta)$ belong to the set

$$
\{-1\} \cup\left\{-\frac{a+b}{d_{0}}\right\} \cup \bigcup_{\left\{\theta \in O_{v} \mid f_{0}\left(1, \theta^{a}\right)=0\right\}} \mathcal{P}\left(\Gamma_{f, \theta}\right) .
$$




\section{Main Result}

In this section we prove the main result of this paper, which gives an explicit list of possible poles of a local zeta function attached to an arithmetically nondegenerate polynomial in terms of the corresponding arithmetic Newton polygon.

6.1. Local zeta functions for arithmetically non-degenerate curves. Let $f(x, y) \in L_{v}[x, y]$ be a non-constant polynomial satisfying $f(0,0)=0$, and

$$
\mathbb{R}_{+}^{2}=\{(0,0)\} \cup \underset{\gamma \subset \Gamma^{\text {geom }}(f)}{\bigcup_{\gamma}} \Delta_{\gamma}
$$

a simplicial conical subdivision subordinated to $\Gamma^{g e o m}(f)$. We denote by $a_{\gamma}=$ $\left(a_{1}(\gamma), a_{2}(\gamma)\right)$ a perpendicular and primitive vector to a facet $\gamma$ of $\Gamma^{\text {geom }}(f)$, and by $\langle a, x\rangle=d_{a}(\gamma)$ the equation of the corresponding supporting line. We also define

$$
\mathcal{P}\left(\Gamma^{\text {geom }}(f)\right):=\left\{-\frac{a_{1}(\gamma)+a_{2}(\gamma)}{d_{a}(\gamma)} \mid \gamma \text { a facet of } \Gamma^{\text {geom }}(f), \text { with } d_{a}(\gamma) \neq 0\right\} .
$$

The following is the main result of the present paper.

Theorem 6.1 (Main Theorem). Let $f(x, y) \in L_{v}[x, y]$ be a non-constant polynomial. If $f(x, y)$ is arithmetically non-degenerate with respect to its arithmetic Newton polygon $\Gamma^{A}(f)$, then the real parts of the poles of $Z(s, f, v)$ belong to the set

$$
\{-1\} \cup \mathcal{P}\left(\Gamma^{g e o m}(f)\right) \cup \mathcal{P}\left(\Gamma^{A}(f)\right)
$$

Proof. By taking a simplicial conical subdivision, the computation of $Z(s, f, v)$ is reduced to the computation of integrals of type $Z\left(s, f, v, O_{v}^{\times 2}\right)$ and $Z\left(s, f, v, \Delta_{\gamma}\right)$, with $\gamma$ a proper face of $\Gamma^{g e o m}(f)$ (see Subsection 2.2). By Lemma 2.1 the real parts of the poles of $Z\left(s, f, v, O_{v}^{\times 2}\right)$ belong to the set $\{-1\}$. The computation of the integrals $Z\left(s, f, v, \Delta_{\gamma}\right)$, for $\gamma$ a proper face of $\Gamma^{\text {geom }}(f)$, involves two cases, according to whether the semi-quasihomogeneous polynomial $f(x, y)$, with respect to $a_{\gamma}=\left(a_{1}(\gamma), a_{2}(\gamma)\right)$, is geometrically non-degenerate or not. If $\Delta_{\gamma}$ is a onedimensional cone, and $f_{\gamma}(x, y)$ does not have singularities on $\left(L_{v}^{\times}\right)^{2}$, then the real parts of the poles of $Z\left(s, f, v, \Delta_{\gamma}\right)$ belong to the set

$$
\{-1\} \cup\left\{-\frac{a+b}{d_{\gamma}}\right\} \subseteq\{-1\} \cup \mathcal{P}\left(\Gamma^{\text {geom }}(f)\right),
$$

where $a x+b y=d_{\gamma}, d_{\gamma} \neq 0$, is the equation of the supporting line of the facet $\gamma$ (cf. Lemma 2.2). If $\Delta_{\gamma}$ is a two-dimensional cone, $f_{\gamma}(x, y)$ is a monomial, and then it does not have singularities on the torus $\left(L_{v}^{\times}\right)^{2}$; by Lemma $2.2 Z\left(s, f, v, \Delta_{\gamma}\right)$ is an entire function.

If $\Delta_{\gamma}$ is a one-dimensional cone, and $f_{\gamma}(x, y)$ has singularities on $\left(L_{v}^{\times}\right)^{2}$, then $f(x, y)$ is a semi-quasihomogeneous arithmetically non-degenerate polynomial, and thus the real parts of the poles of $Z\left(s, f, v, \Delta_{\gamma}\right)$ belong to the set

$$
\{-1\} \cup\left\{-\frac{a+b}{d_{\gamma}}\right\} \cup \mathcal{P}\left(\Gamma^{A}(f)\right) \subseteq\{-1\} \cup \mathcal{P}\left(\Gamma^{g e o m}(f)\right) \cup \mathcal{P}\left(\Gamma^{A}(f)\right),
$$

(cf. Theorem 5.1). Therefore the real parts of the poles of $Z(s, f, v)$ belong to the set $\{-1\} \cup \mathcal{P}\left(\Gamma^{\text {geom }}(f)\right) \cup \mathcal{P}\left(\Gamma^{A}(f)\right)$. 


\section{REFERENCES}

[1] Arnold V., Varchenko A. and Gussein-Zade S., Singularités des applications différentiables, vol. 2, Mir, Moscow, 1986.

[2] Denef J., Report on Igusa's local zeta function, Seminaire Bourbaki 1990/1991 (730-744) in Asterisque 201-203 (1991), 359-386. MR 93g:11119

[3] Denef J., Poles of $p$-adic complex powers and Newton polyhedra, Nieuw Archief voor Wiskunde 13 (1995), 289-295. MR 96m:11106

[4] Denef J., Hoornaert Kathleen, Newton polyhedra and Igusa local zeta function, J. Number Theory 89 (2001), no. 1, 31-64. MR 2002g:11170

[5] Igusa Jun-Ichi, An introduction to the theory of local zeta functions, AMS/IP studies in advanced mathematics, v. 14, 2000. MR 2001j:11112

[6] Igusa Jung-Ichi, A stationary phase formula for $p$-adic integrals and its applications, in Algebraic Geometry and its Applications, Springer-Verlag, New York, 1994, pp. 175-194. MR 95a:11104

[7] Igusa Jung-Ichi, Complex powers of irreducible algebroid curves, Geometry Today, Birkhäuser, Boston, 1985, pp. 207-230. MR 88j:11084

[8] Kempf G., Knudsen F., Mumford D., and Saint-Donat B., Toroidal Embeddings, Lecture Notes in Mathematics, vol. 339, Springer-Verlag, Berlin, 1973. MR 49:299

[9] Kouchnirenko A. G., Polyèdres de Newton et nombres de Milnor, Invent. Math. 32 (1976), 1-31. MR 54:7454.

[10] Lichtin B., Meuser D., Poles of a local zeta function and Newton polygons, Compos. Math. 55 (1985), 313-332. MR 87a:11120

[11] Meuser D., On the poles of a local zeta function for curves, Invent. Math. 73 (1983), 445-465. MR 85i: 14014

[12] Strauss L., Poles of two-variable p-adic complex power, Trans. Amer. Math. Soc. 27 (1983), 481-493. MR 84k:14019

[13] Varchenko A., Newton polyhedra and estimation of oscillanting integrals, Funct. Anal. Appl. 10 (1976), 175-196.

[14] Veys W., On the poles of Igusa's local zeta functions for curves, J. Lond. Math. Soc. 41 (1990), 27-32. MR 92j:11142

[15] Veys W., Poles of Igusa's local zeta function and monodromy, Bull. Soc. Math. Fr. 121 (1993), 545-598. MR 95b:11110

[16] Zuniga-Galindo W. A., Igusa's local zeta functions of semiquasihomogeneous polynomials, Trans. Amer. Math. Soc. 353 (2001), 3193-3207. MR 2001j:11116

[17] Zuniga-Galindo W. A., Local zeta functions and Newton polyhedra, to appear in Nagoya Math. J.

[18] Zuniga-Galindo W. A., Local zeta function for polynomial non-degenerate homogeneous mappings, preprint 2003.

Instituto de Matemática E Computaçao, Universidade de São Paulo at São Carlos, Av. do Trabalhador São-Carlense 400, CEP 13560-970, São Carlos - SP, Brasil

E-mail address: mjsaia@icmc.usp.br

Department of Mathematics and Computer Science, Barry University, 11300 N.E. Second Avenue, Miami Shores, Florida 33161

E-mail address: wzuniga@mail.barry.edu 